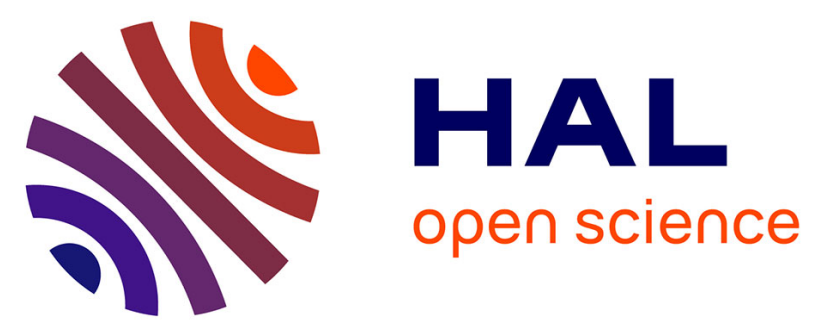

\title{
Perceived unfairness of revenue management pricing: developing a measurement scale in the context of hospitality
}

\author{
Sourou Meatchi, Sandra Camus, Danielle Lecointre-Erickson
}

\section{To cite this version:}

Sourou Meatchi, Sandra Camus, Danielle Lecointre-Erickson. Perceived unfairness of revenue management pricing: developing a measurement scale in the context of hospitality. International Journal of Contemporary Hospitality Management, 2021, ahead-of-print (ahead-of-print), pp.109 - 131. 10.1108/IJCHM-11-2020-1344 . hal-03373504

\author{
HAL Id: hal-03373504 \\ https://hal.science/hal-03373504
}

Submitted on 11 Oct 2021

HAL is a multi-disciplinary open access archive for the deposit and dissemination of scientific research documents, whether they are published or not. The documents may come from teaching and research institutions in France or abroad, or from public or private research centers.
L'archive ouverte pluridisciplinaire HAL, est destinée au dépôt et à la diffusion de documents scientifiques de niveau recherche, publiés ou non, émanant des établissements d'enseignement et de recherche français ou étrangers, des laboratoires publics ou privés. 
Recherche et Applications en Marketing

Stratégie de tarification par le

(C) l'Association Française du Marketing, 2020

Article reuse guidelines:

revenue management dans le secteur sagepub.com/journals-permissions hôtelier: réduire l'injustice perçue pour favoriser le consentement à payer

\title{
Sourou MEATCHID
}

Université d'Angers, UFR Esthua Tourisme et Culture

GRANEM (Groupe de Recherche ANgevin en Economie et Management)

\section{Sandra CAMUS}

Université d'Angers, UFR Esthua Tourisme et Culture

GRANEM (Groupe de Recherche ANgevin en Economie et Management)

\begin{abstract}
Résumé
Dans un contexte de concurrence toujours exacerbée, la tarification par le revenue management (TRM) est devenue un outil stratégique pour les entreprises disposant de capacités contraintes. Cependant, en dépit de son intérêt considérable, des études montrent que la TRM fait l'objet de réactions mitigées de la part des consommateurs. L'objectif de cette recherche est de tester des leviers d'actions susceptibles de contribuer à réduire l'injustice perçue à l'égard de la TRM et ainsi, favoriser le consentement à payer (CAP). Deux échantillons quantitatifs $(\mathrm{NI}=325 ; \mathrm{N} 2=280)$ ont permis de valider les instruments de mesure des concepts mobilisés et tester deux modèles explicatifs de "fairness based pricing". Les résultats montrent que l'équité et la transparence ont des effets individuels et d'interaction fort positifs sur la réduction des dimensions cognitives de l'injustice perçue et sur le renforcement du CAP. En revanche, les effets sur les dimensions affectives de l'injustice perçue ne sont pas validées dans les deux modèles testés.
\end{abstract}

\section{Mots-clés}

Consentement à payer (CAP), équité perçue, injustice perçue, pricing, revenue management, transparence perçue, yield management

\section{Introduction générale}

Dans une économie de marché caractérisée par des logiques concurrentielles, la performance des entreprises passe inévitablement par des innovations de produits ou d'approches marketing. Parmi les innovations marketing développées dans le secteur des services au cours de ces dernières décennies, on note la pratique du revenue management (RM). Connu à l'origine sous le nom de yield management, le RM (annexe 1) s'est progressivement enrichi par de nouveaux leviers d'optimisation des quantités

\footnotetext{
Auteur correspondant :

Sourou Meatchi, Université d'Angers, UFR Esthua Tourisme et Culture, GRANEM (Groupe de Recherche ANgevin en Economie et Management), 5 rue des ardrières, 49100 Angers, Angers, 49035, France

Email: sourou.meatchi@univ-angers.fr
} 
d'offres de services et des prix pour devenir une stratégie globale de gestion dans les entreprises caractérisées par des actifs périssables et soumises à une demande erratique (Domingo-Carrillo et al., 2019; Weatherford et Bodily, 1992). Le RM repose sur une connaissance précise du comportement du consommateur, une segmentation très fine de la demande et une modulation en temps réel des capacités (offres) dans le but d'allouer le bon prix au bon client et au bon moment (Abrate et al., 2019). Depuis les années 1980, la pratique du RM a favorisé l'émergence d'une nouvelle approche de prix dans le secteur des services. Ce nouveau paradigme tarifaire, dénommé le pricing ou la tarification par le revenue management (TRM), s'est renforcé à la suite du développement des technologies de l'information et plus particulièrement de l'Internet (Noone, 2016; Vives et al., 2018). Avec le développement des techniques de l'intelligence artificielle (algorithmique, machine learning, sciences cognitives, etc.), la pratique de la TRM est conduite à se renforcer et à se généraliser au sein des entreprises de services (transports, hôtellerie, restaurants, etc.). La TRM permet aux entreprises de services d'optimiser leurs revenus. Pour le consommateur, le prix est une variable décisive dans le processus de choix de services (séjour en hôtel, visite d'un parc à thème, location d'une voiture, etc.). Cependant, les prix basés sur la TRM font l'objet d'une perception mitigée. Certains consommateurs les jugent tout à fait équitables alors que d'autres les trouvent inacceptables. En effet, pour un même trajet et sur le même créneau horaire, les prix d'un billet d'avion ou de train peuvent être très différents d'un client à un autre. De même, réserver une chambre d'hôtel longtemps à l'avance (early booking) ne suffit pas toujours pour obtenir des prix avantageux par rapport aux clients qui réservent à la dernière minute et qui peuvent obtenir des prix «cassés». Face à ce dilemme, le consommateur se trouve dans une perplexité qui le conduit souvent à avoir une image négative de la TRM. Pour Camus et al. (2014), quel que soit le prix payé par le client (inférieur ou supérieur au prix espéré), les risques d'injustice perçue à l'égard de la TRM sont élevés. Dans le cas d'un prix désavantageux, le client peut avoir des difficultés à accepter qu'il aurait pu payer moins cher le même service. Dans le cas d'un prix avantageux, malgré une valeur perçue positive, le prix basé sur le RM peut également être considéré comme injuste à cause de son caractère discriminatoire. L'injustice perçue est à l'évidence un risque permanent pour les entreprises qui pratiquent la TRM. Au regard du constat dressé ci-dessus, la question centrale de cette recherche est de comprendre comment se manifestent les perceptions d'injustice à l'égard de la TRM et quels sont les leviers pour réduire ces perceptions et pour favoriser le consentement des consommateurs à payer. Les réponses à ces questions permettent d'apporter plusieurs contributions théoriques et managériales. Sur le plan théorique, notre recherche répond à l'appel de plusieurs auteurs (ex. : Colquitt et al., 2015; Kimes et Wirtz, 2015; Rupp et al., 2017) qui encouragent les chercheurs à étudier davantage le concept d'injustice perçue car selon eux, l'injustice est très saillante pour toute personne qui en a déjà fait l'expérience. Ensuite, notre recherche vise à apporter un éclairage théorique et empirique sur les effets de l'équité et de la transparence sur la perception et l'acceptabilité des prix découlant de la TRM. Dans cette optique, une modélisation de fairness-based pricing ou «tarification basée sur la justice» sera proposée et discutée. Pour cette modélisation, nous nous appuierons dans un premier temps sur la littérature dominante (ex.: Kahneman et al., 1986; Sahut et al., 2016; Xia et al., 2004) qui conduit à positionner la transparence perçue et l'équité perçue comme deux variables explicatives indépendantes l'une de l'autre dans leurs relations avec l'injustice perçue et avec le CAP (Modèle 1). Dans un second temps, nous mobiliserons les autres approches (ex. : Maxwell, 2008; Noon, 2016; Zhang et Jiang, 2014) qui amènent à s'interroger sur un éventuel rôle modérateur de la transparence perçue sur la relation entre l'équité perçue et l'injustice perçue, d'une part, et sur la relation entre cette même variable indépendante et le CAP, d'autre part (Modèle 2). Notre recherche vise donc à tester et à comparer deux modèles (un modèle à deux variables indépendantes et un modèle à une variable indépendante et une variable modératrice) afin de connaître leur performance en matière de réduction de l'injustice perçue et du renforcement de consentement à payer les prix basés sur la TRM. Du point de vue managérial, cette recherche devrait 
aider les entreprises de services à mieux comprendre le phénomène de l'injustice perçue à l'égard de la TRM. Elle devrait aussi leur fournir de nouveaux leviers stratégiques et opérationnels permettant de réduire ce phénomène et ainsi favoriser le consentement à payer (CAP). L'article est structuré en quatre grandes parties. La première présente la revue de littérature et ses limites. La deuxième porte sur le modèle et les hypothèses de recherche. La troisième décrit la méthodologie mise en œuvre pour collecter et analyser les données empiriques. Enfin, la quatrième partie expose les résultats de la recherche. A la suite des quatre parties, une conclusion générale permet de discuter les résultats et de suggérer des voies de recherche futures.

\section{Etat de l'art sur la perception et le consentement à payer des prix basés sur la TRM: une littérature riche mais insuffisante}

Cet article s'inscrit dans la lignée de la littérature sur la perception des prix basés sur la TRM. La perception du prix peut être définie comme un jugement porté par le consommateur à l'égard d'un montant monétaire qu'il doit débourser pour acquérir un produit. Ce jugement peut être positif (justice perçue) ou négatif (injustice perçue) et conduit le consommateur à accepter ou à refuser la transaction (Bolton et al., 2003; Lu et al., 2019). La question de la justice du prix est de tous les temps un sujet d'actualité car elle préoccupe quotidiennement la plupart des consommateurs. En effet, que ce soit le prix de l'essence, les frais médicaux ou encore la tarification dynamique d'amazon.com, la plupart des actes de consommation sont liés à un prix. Cependant, malgré leur importance dans les systèmes de transaction, les prix posent souvent des problèmes de justice. Par exemple, lorsqu'un client d'amazon.com découvre que le prix d'un même produit (un $\mathrm{CD}$, un livre, un jouet, etc.) varie d'un moment à un autre ou d'un contexte à un autre, il peut se mettre en colère contre l'entreprise (Adamy, 2000; Tripathi, 2017). Cet exemple montre la manière dont les prix et en particulier, ceux qui sont fondés sur le RM, peuvent conduire à une perception d'injustice et avoir des conséquences dommageables pour les entreprises. La littérature postule par ailleurs que l'injustice perçue constitue la cause principale des baisses du consentement à payer des prix découlant de la TRM (Noone et Mattila, 2009; Wu et al., 2012). En dépit de la richesse de la littérature disponible, il existe peu d'études empiriques sur la manière dont les perceptions et l'acceptabilité de la TRM pourraient être améliorées. Il est donc important d'explorer de nouvelles pistes de recherche sur les stratégies à mettre en œuvre afin de limiter les risques d'injustice perçue et ses corolaires dans le contexte de la TRM.

\section{Cadre théorique et conceptuel de la recherche}

Comme souligné supra, malgré une littérature foisonnante, force est de constater que peu de modèles empiriques ont déjà été testés sur les stratégies de réduction de l'injustice perçue et sur le consentement à payer des prix découlant de la TRM. Le dessein de cette recherche est de contribuer à combler cette lacune en proposant et comparant deux modèles de tarification basée sur la justice (Fairness-based pricing). Ces deux modèles s'appuient sur la théorie de la justice des prix. Formulée par Xia et al. (2004) à partir des travaux sur le principe de l'intérêt mutuel (Kahneman et al., 1986) et sur les théories de la justice sociale (Adams, 1965) et organisationnelle (Greenberg, 1987), la théorie de la justice des prix analyse la manière dont les consommateurs évaluent les prix et les traitements dont ils font l'objet dans le cadre des relations transactionnelles. Cette théorie psychoéconomique met en évidence deux facteurs de justice: l'équité perçue d'une part, et la transparence perçue, d'autre part. Selon Xia et al. (2004), les deux facteurs sont très importants pour les consommateurs parce qu'ils sont dans leur intérêt. $\mathrm{Si}$ un consommateur a le sentiment que sa contribution dans une transaction n'est pas récompensée à sa juste valeur, il peut ressentir de l'injustice. De même, un consommateur peut ressentir de l'injustice si les informations mises à sa disposition ne sont pas transparentes (Campbell, 2007). Par ailleurs, la littérature postule que dans le contexte de la TRM, l'absence d'équité et de 
transparence perçues conduit généralement à une baisse du CAP (Kimes et Wirtz, 2016; Noone et Mattila, 2009).

Avant d'analyser les relations de cause à effet qui sous-tendent nos deux modèles de recherche, il convient de présenter chacun des concepts mobilisés grâce à la revue de littérature et en particulier, grâce à l'analyse de la théorie de la justice des prix.

L'équité perçue du prix (EPP). Dans le contexte de prix, le principe d'équité (Deutsch, 1975; Xia et al., 2004) considère que dans les relations d'échange, la justice est respectée lorsque le ratio coûts/bénéfices est équilibré. Ce principe postule également que la justice consiste à donner à tous les consommateurs les mêmes chances d'accès à un produit ou à un prix. Le modèle de Deutsch (1975) suggère que dans le contexte des échanges transactionnels, c'est le principe d'équité (ratio coûts/bénéfices) qui doit être pris en compte. Cette approche de la justice distributive a été adoptée dans de nombreuses recherches sur les prix (ex.: Inman et Nikolova, 2017 ; Taylor et Kimes, 2011). Dans cette recherche, nous mobiliserons le concept d'équité au sens de Deutsch (1975) mais aussi au sens d'Oliver et Swan (1989a, b), Xia et al. (2004) et de Vukadin et al., (2019).

La transparence perçue de l'information (TPI). Audelà de l'équité, la transparence joue également un rôle important dans le jugement des prix (Noone, 2016; Sahut et al., 2016). Selon Heyman et Mellers (2008), dans le cadre d'un processus d'achat, le consommateur évalue non seulement le niveau des prix mais aussi la transparence des informations sur le rapport qualité/prix, les conditions de vente et les avantages liés à chaque niveau de prix. Pour cela, il s'appuie généralement sur l'information mise à sa disposition par l'entreprise et le cas échéant, par d'autres parties prenantes (autres clients, associations de consommateurs, administrations publiques, etc.). Si l'information disponible ne permet pas de comprendre la politique tarifaire de l'entreprise, le consommateur peut ressentir de l'injustice. La transparence de l'information repose sur la clarté, la précision, la cohérence et la fiabilité des éléments communiqués (Colquitt et al., 2015). De nombreux auteurs (ex. : Choi et Mattila, 2005; Ferguson et al.,
2014) montrent que l'absence ou l'insuffisance d'information sur les prix peut conduire le consommateur à douter de l'équité du prix. En revanche, le fait de fournir des justifications concernant la politique tarifaire réduirait les jugements négatifs et favoriserait le CAP (Bearden et al., 2003; Li et al., 2019).

L'injustice perçue à l'égard de la TRM. Dans le contexte de la TRM, l'injustice perçue est généralement définie comme une perception négative de la valeur d'une transaction (Camus et al., 2014). Dans le secteur de l'hôtellerie, certaines études ont montré qu'un client qui paie plus cher qu'un autre pour un service similaire sans apercevoir une différence dans les attributs du produit peut juger la situation comme étant injuste (Kimes, 1994). Pour Xia et al. (2004), l'injustice à l'égard du prix comporte deux dimensions: l'une cognitive et l'autre affective. La dimension cognitive indique que les perceptions d'injustice reposent sur la comparaison avec un standard pertinent (par exemple, le prix moyen du marché), une référence (par exemple, le prix d'un précédent achat) ou une norme (par exemple, le tarif-adhérent). La dimension affective quant à elle, se traduit par des émotions négatives qui accompagnent la cognition. Les deux dimensions seront mobilisées dans cette recherche.

Le consentement à payer les prix découlant de la TRM. Dans les recherches en comportement du consommateur, le consentement à payer (CAP) est généralement défini comme l'attitude probable qu'un consommateur peut adopter à l'égard d'un prix (Dodds et al., 1991; Tanford et al., 2018). Selon Le Gall-Ely (2009), le CAP s'inscrit dans le processus de perception des prix et se rapproche des concepts de prix de référence et de prix acceptables. Il est aussi lié à d'autres variables influençant le processus de décision (satisfaction, fidélité, etc.). Dans les approches perceptuelles, le CAP sert à mesurer soit l'acceptabilité d'un prix précis pour un produit donné (par exemple, consentement à payer $2 €$ pour une petite bouteille d'eau dans une gare; consentement à payer $60 €$ ou $150 €$ pour un vol Paris-Nice), soit l'acceptabilité d'une famille de prix (par exemple, consentement à payer les prix dynamiques des compagnies aériennes). Dans cette 
recherche, c'est le consentement à payer une famille ou un modèle de prix qui sera privilégié. Ce choix s'explique par le fait que notre étude est orientée vers la mesure de l'acceptabilité d'un modèle tarifaire (en l'occurrence, la TRM) et non pas vers la mesure de l'acceptabilité d'un montant précis (par exemple, consentement à payer un prix de $950 €$ pour un smartphone). Dans cette optique, c'est la mesure par des items qualitatifs ou sémantiques qui sera utilisée. Cette approche a déjà été utilisée par d'autres chercheurs en comportement du consommateur. Par exemple, dans son étude sur le consentement à payer les prix des billets d'avion, Maxwell (2002) a mesuré le CAP avec les indicateurs sémantiques (annexe 2). Dans le secteur hôtelier, Noone et Mattila (2009) ont adapté l'échelle sémantique de Grewal et al. (1998) pour mesurer le consentement à payer les prix variables des chambres d'hôtel.

\section{Les hypothèses de recherche des deux modèles comparés}

Sur la base de la littérature, nous proposons deux modèles de «tarification basée sur la justice» ou fairness-based pricing. Rappelons que selon la théorie de la justice des prix (Xia et al., 2004), l'équité perçue et la transparence perçue auraient des effets positifs sur la perception des prix. Malgré ce postulat, aucun modèle empirique n'a encore été testé sur les effets de l'équité perçue et de la transparence perçue sur la réduction de l'injustice perçue et sur le CAP dans le contexte de la TRM pratiquée par les hôtels. Ensuite, aucune recherche à notre connaissance ne s'est encore intéressée aux effets d'interactions entre l'équité perçue et la transparence perçue dans le contexte de la TRM. Pourtant, l'analyse de la littérature laisse penser que l'effet de l'équité sur l'injustice perçue dépend de la politique de transparence de l'information (Maxwell, 2008). Il apparait donc que l'équité perçue du prix et la transparence perçue de l'information pourraient avoir, d'un côté, des effets individuels et directs (modèle 1) et, de l'autre côté, des effets d'interactions avec l'équité perçue comme variable indépendante et la transparence perçue comme variable modératrice (modèle 2). Nous testerons et comparerons les deux modèles afin de savoir lequel donne les meilleurs résultats en matière de réduction d'injustice perçue et en matière du renforcement du CAP. Dans cette perspective, nous présentons dans un premier temps, les hypothèses sur les effets individuels et directs de l'équité perçue et de la transparence perçue sur la réduction de l'injustice perçue et sur le CAP (modèle 1). Dans un second temps, les hypothèses sur les effets d'interactions entre l'équité perçue (variable indépendante) et la transparence (variable modératrice) sont formulées (modèle 2).

\section{Effets individuels de l'équité perçue et de la transparence perçue sur l'injustice perçue et sur le consentement à payer (modèle I - Figure I)}

Selon les approches heuristiques de la justice sociale (ex.: Brown-Liburd et al., 2018; Lind, 2001), en situation d'incertitude, les individus prennent des décisions soit en se limitant à la première solution identifiée soit en procédant par des approches séquentielles permettant d'éliminer progressivement les alternatives défavorables pour ne conserver qu'une gamme restreinte de solutions perçues comme optimales. Les comportements heuristiques des consommateurs peuvent donc les conduire à faire des «raccourcis» de jugements en se limitant aux premiers éléments (par exemple, le ratio coûts/bénéfices, l'information disponible, les procédures, etc.) qu'ils identifient. Dans ces conditions, le consommateur n'aurait pas besoin d'obtenir simultanément l'équité et la transparence pour apprécier la justice ou l'injustice d'un prix. La réduction de l'injustice perçue et le CAP pourraient dès lors être favorisés par la présence d'un seul facteur principal. Ce facteur principal peut être l'équité perçue du prix ou la transparence perçue de l'information. Les clients dont le critère le plus important est l'équité se contenteront de l'équilibre du ratio coûts/bénéfices pour apprécier la justice d'un prix. Ceux qui sont sensibles à la transparence porteront leur attention sur la quantité et la qualité des informations mises à leur disposition (Miao et Mattila, 2007). Nous présentons et justifions ci-après, les hypothèses selon lesquelles l'équité perçue du prix (EPP) et la transparence perçue de l'information (TPI) auraient chacune des effets 


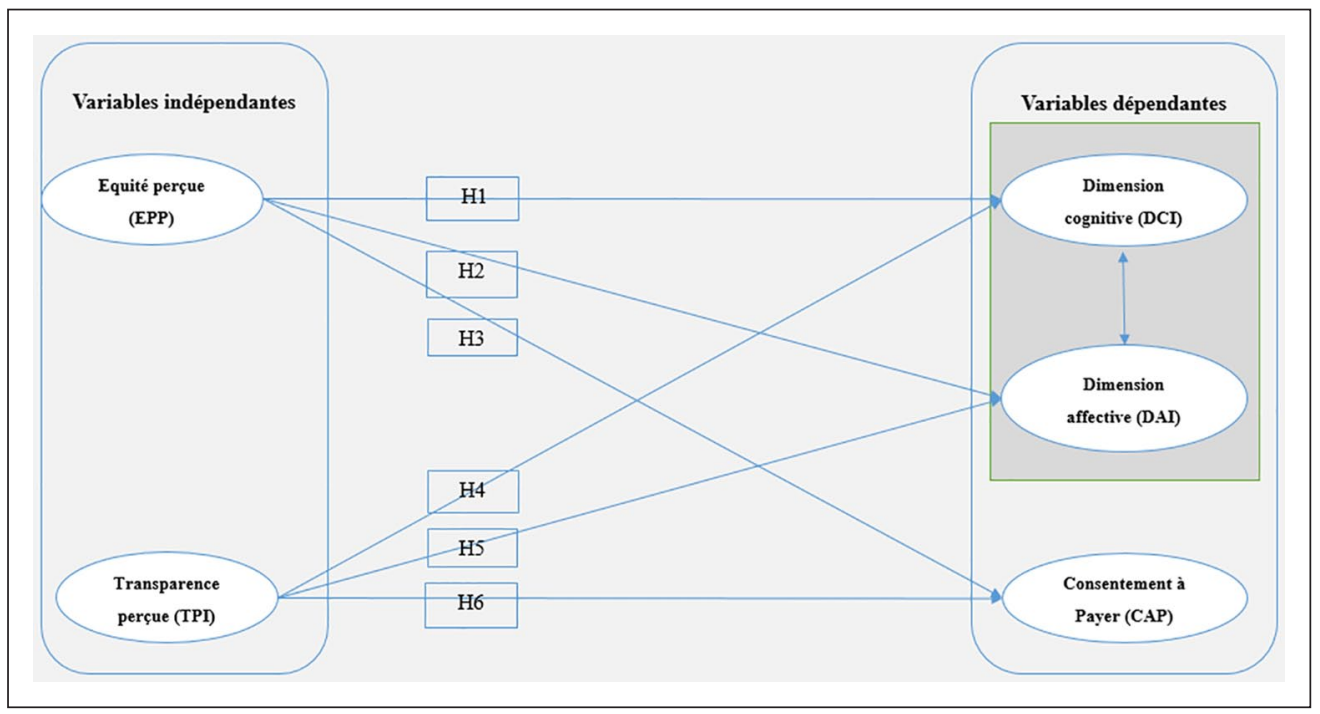

Figure I. Effets individuels de l'équité perçue et de la transparence perçue sur l'injustice perçue et sur le CAP (modèle I)

individuels positifs sur la réduction de l'injustice perçue et sur le consentement à payer dans le contexte de la TRM.

Effets de l'équité perçue sur la réduction de l'injustice perçue et sur le CAP. De nombreuses recherches antérieures postulent que l'absence d'équité est un facteur des perceptions d'injustice à l'égard de la TRM. Selon Kimes (1994) et Kimes et Wirtz (2016), l'application du principe du dual entitlement (Kahneman et al., 1986) dans le contexte des prix permet de considérer que la TRM est injuste. Ceci, par ce que les prix basés sur la TRM ne sont pas toujours liés aux coûts de production mais à l'exploitation des anomalies économiques du marché. Pour Campbell (2007), les consommateurs ressentent de l'injustice à l'égard des prix qui ne sont pas justifiés par des motifs valables. En revanche, lorsque le consommateur trouve son intérêt dans une politique de prix, il devient moins exigeant en matière de justice tarifaire (Camus et al., 2014; Kimes et Wirtz, 2002). On constate à travers la littérature disponible que certains auteurs (ex.: Bolton et al., 2003) se focalisent principalement sur l'analyse du rôle de l'équité dans la perception des prix et du CAP. Au regard des éléments qui viennent d'être évoqués, il semblerait, toutes choses égales par ailleurs, que l'équité du prix peut suffire à atténuer l'injustice perçue et à favoriser le CAP. De ce postulat découlent nos trois premières hypothèses de recherche qui sont les suivantes:

H1. L'équité perçue a un effet positif sur la réduction de la dimension cognitive de l'injustice perçue à l'égard de la TRM

H2. L'équité perçue a un effet positif sur la réduction de la dimension affective de l'injustice perçue à l'égard de la TRM

H3. L'équité perçue a un effet positif sur le consentement à payer les prix découlant de la TRM.

Effets de la transparence perçue sur la réduction de l'injustice perçue et sur le CAP. Concernant les effets individuels de la transparence perçue, Miao et Mattilla (2007) postulent que la qualité des informations disponibles joue un rôle fondamental dans la perception des prix. Pour leur part, Morwitz et al. (1998) suggèrent que la manière dont les prix sont présentés (affichage complexe versus affichage simple) est un antécédent de la transparence perçue qui est, elle-même, un facteur de la justice perçue et du CAP. Il a aussi été démontré que les comportements heuristiques des consommateurs les conduisent souvent à faire des jugements rapides en 
se limitant aux critères élémentaires qui sont à leur disposition (Lind, 1992, 2001). Les consommateurs dont le critère le plus important est la transparence se limiteront à cet indicateur dans leur processus d'évaluation de la justice d'un prix. Ils peuvent s'intéresser à la clarté, à la cohérence et à la fiabilité de l'information afin de savoir si le prix est juste (Maxwell, 2008; Tanford, Baloglu et Erdem, 2012). Dans ces conditions, lorsque l'individu ne dispose pas d'informations transparentes lui permettant d'évaluer ses avantages au regard de ses coûts (équité interne) ou d'apprécier le ratio couts/bénéfices des autres clients (équité externe), il peut considérer que le prix payé ou observé est injuste. Pour atténuer le sentiment d'injustice perçue et ses conséquences sur le CAP, certains auteurs (Campbell, 2007; Li, Li et Wang, 2019) suggèrent d'utiliser la transparence de l'information car cette variable jouerait un rôle individuel important dans la perception des prix. En nous appuyant sur les éléments de la littérature relative à la transparence des prix, nous proposons les hypothèses suivantes:

H4. La transparence perçue a un effet positif sur la réduction de la dimension cognitive de l'injustice perçue à l'égard de la TRM.

H5. La transparence perçue a un effet positif sur la réduction de la dimension affective de l'injustice perçue à l'égard de la TRM.

H6. La transparence perçue a un effet positif sur le consentement à payer les prix découlant de la TRM.

\section{Effets d'interaction de l'équité perçue et de la transparence perçue sur l'injustice perçue et sur le CAP (modèle 2 - Figure 2)}

Comme nous l'avons déjà vu à travers la revue de la littérature, dans les modèles sur la perception des prix, les effets de l'équité perçue et ceux de la transparence perçue sont généralement étudiés de façon séparée ou séquentielle. En revanche, aucun modèle à notre connaissance n'a empiriquement testé les effets d'interactions de l'équité perçue et de la transparence perçue sur la réduction de l'injustice perçue et sur le CAP. Or certaines recherches (ex. : Maxwell, 2008; Sahut et al., 2016) laissent penser que pour réduire l'injustice perçue à l'égard de la TRM et favoriser le CAP, toute démarche d'équité doit être accompagnée par une politique de transparence de l'information. En effet, selon Maxwell (2008), une politique d'équité qui n'est pas accompagnée d'une information transparente est inefficiente pour atténuer les perceptions d'injustice et pour favoriser le consentement à payer. Dans le contexte de la TRM, l'équité perçue et la transparence perçue produiraient donc des effets d'interactions positifs en matière de réduction de l'injustice perçue et en matière de CAP. Au regard de ce qui précède, nous supposons qu'il existe des effets d'interaction entre l'équité perçue du prix et la transparence perçue de l'information sur la réduction de l'injustice perçue et sur le CAP. Ce postulat nous amène à formuler les hypothèses suivantes:

H7. L'équité perçue et la transparence perçue ont des effets d'interactions positifs sur la réduction de la dimension cognitive de l'injustice perçue à l'égard de la TRM.

H8. L'équité perçue et la transparence perçue ont des effets d'interactions positifs sur la réduction de dimension affective de l'injustice perçue à l'égard de la TRM.

H9. L'équité perçue et la transparence perçue ont des effets d'interactions positifs sur le consentement à payer les prix découlant de la TRM.

Dans ce second modèle (Figure 2), l'équité perçue garde son statut de variable indépendante alors que la transparence perçue devient une variable modératrice. Cette nouvelle répartition de rôle entre les deux variables se fonde sur les approches distributives (Oliver et Swan, 1989; Xia et Monroe, 2010 ) et heuristiques de la justice des prix (BrownLiburd et al., 2018; Maxwell, 2008; Lind, 2001). Ces différentes approches ont montré que dans le contexte des prix, les clients ont tendance à considérer en priorité les critères d'équité. Selon Maxwell (2008), le premier élément que le consommateur évalue dans le contexte des prix est l'équité. Si le prix observé ou obtenu est différent 


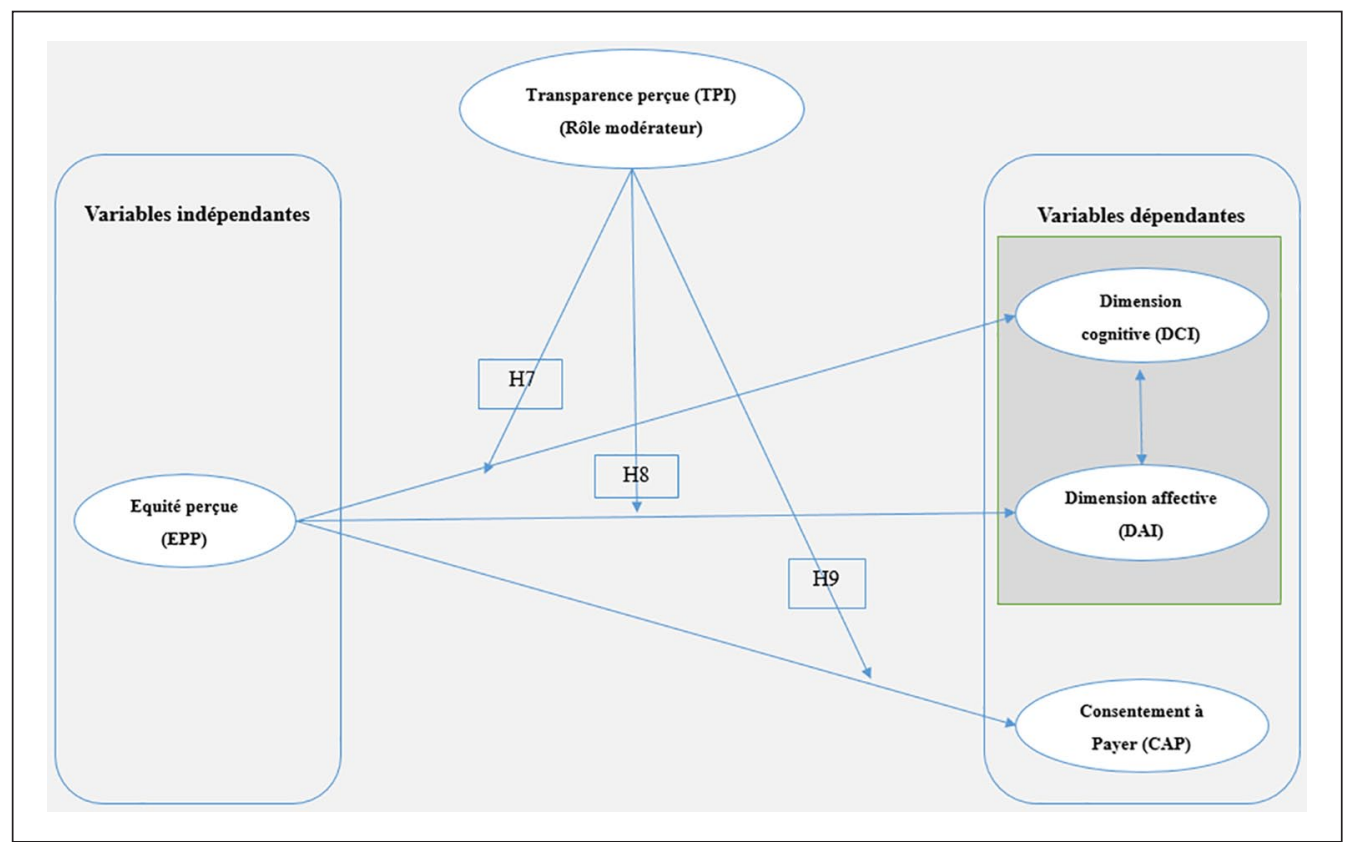

Figure 2. Rôle modérateur de la transparence perçue sur la relation entre l'équité perçue et l'injustice perçue et le CAP (modèle 2)

du prix habituel ou du prix payé par les autres clients, le consommateur procède alors à la recherche des raisons qui justifient les différences constatées entre les prix. Dans ces conditions, la transparence de l'information sur les prix devient un modérateur des effets de l'équité perçue sur l'injustice perçue et sur le CAP.

\section{Méthodologie de la recherche}

Dans cette partie, nous présentons la méthodologie de collecte et d'analyse de données puis le processus de choix et de validation des instruments de mesure.

Collecte et pré analyses des données quantitative. Dans le but d'opérationnaliser les construits et de tester le modèle de structure, nous avons effectué deux collectes de données quantitatives. La première $\left(N_{1}=325\right)$ a été réalisée par un questionnaire administré en face-à-face. L'échantillon est très varié en terme d'âge ( $21-25$ ans : $42 \% ; 26-35$ ans : $15 \% ; 36-45$ ans : $20 \% ; 46$ ans et plus : $23 \%$ ), de sexe (Femmes : 62\%; Hommes : 37\%; Autres : 1\%) et de catégorie socioprofessionnelle (PCS + : $23 \%$; PCS-: 29\%; Retraités: 4\%, Etudiants/doctorants :
24\% ; Autres : 20\%). Cette première collecte a permis de pré-tester les échelles de mesure des variables mobilisées. La seconde collecte $\left(N_{2}=280\right)$ a été menée au moyen d'une méthode expérimentale basée sur des scénarios (Bolton et al., 2003; Lavorata, Nillès et Pontier, 2005). Un plan factoriel 2X2 (annexe 4) a été mis en place à cet effet. Les scénarios expérimentaux ont été construits avec l'aide de trois hôteliers de la ville d'Angers (Hôtel d'Anjou 4*, Hôtel Les Trois Lieux 3*, Hôtel Iéna $2^{*}$ ). Les personnes interrogées ont été affectées de façon aléatoire dans les quatre conditions expérimentales. Chaque sujet est affecté dans un seul groupe expérimental. Il s'agit donc d'une expérimentation intersujets. La collecte de données a été effectuée par Internet auprès d'un Access Panel de la société d'étude CReATESTS. Elle a permis d'interroger un échantillon total de 280 répondants. Les caractéristiques sociodémographiques de l'échantillon sont relativement variées concernant le sexe (Femmes: 41\%; Hommes: 59\%), d'âge (21-30 ans: 25\%; $31-40: 25 \% ; 41-50: 25 \%$; 51 et plus : $25 \%$ ) et la catégorie socioprofessionnelle (PCS+: 23\%; PCS-: $30 \%$; Professions intermédiaires: 18\%; Retraités : 4\%; Autres : 25\%). 
Validation des instruments de mesure. Les instruments de mesure mobilisés ont été empruntés à la littérature existante. Ils ont tous été soumis aux tests de purification et aux analyses statistiques en suivant le paradigme de Churchill (1979), les recommandations de Rossiter $(2002$; 2011) ainsi que les critères de Fornell et Larcker (1981). Les tests statistiques ont permis de confirmer la fiabilité et la validité des concepts mobilisés (annexe 3).

Mesure de l'injustice perçue à l'égard de la TRM. L'échelle de l'injustice perçue a été empruntée à Meatchi et Camus (2018b). Cette échelle est composée de trois dimensions (déviance normative perçue, opacité perçue et affects négatifs) et de neuf items réflexifs (Jarvis et al., 2003) qui totalisent une variance expliquée de $78,52 \%$. La forte corrélation entre la déviance normative et l'opacité perçue nous a conduits à tester un modèle de second ordre à deux dimensions, à savoir, une dimension cognitive avec cinq items $(\alpha: 0,89 ; p: 0,92 ; A V E: 0,70)$ et une dimension affective avec trois items $(\alpha: 0,74$; $p: 0,85 ; A V E: 0,66)$. Les alphas de Cronbach $(\alpha)$ et les rhôs de Dillon-Goldstein $(p)$ sont satisfaisants car ils ont tous des coefficients supérieurs à 0,07 ; seuil habituellement recommandé dans la littérature en psychométrie. Enfin, cette échelle a été intégrée dans le modèle explicatif, ce qui a permis de tester sa validité prédictive.

Mesures des autres construits du modèle. Les échelles des autres construits (CAP, équité perçue et transparence perçue) ont également été empruntées à la littérature puis testées afin de vérifier leur fiabilité et leur validité. Pour le CAP, nous avons emprunté et adapté l'échelle de Dodds et al. (1991) composée de cinq items. Sur les cinq indicateurs, nous avons retenu les trois les plus adaptés à nos objectifs de recherche. Les tests de fiabilité et de validité sont très satisfaisants $(\alpha=0,74 ;$ rhô $=0,85 ; A V E=$ $0,65)$. Concernant la mesure de l'équité perçue, nous avons emprunté trois items de l'échelle d'Oliver et Swan (1989). Les résultats des tests statistiques sont également très satisfaisants $(\alpha=0,81$; $r h \hat{o}=0,88 ; A V E=0,71)$. Enfin, pour mesurer la transparence perçue, ce sont les indicateurs de la justice informationnelle (Colquit et al., 2015) qui ont été privilégiés. Trois indicateurs de mesure de la transparence informationnelle (clarté, explication, précision) ont été retenus et testés. Les résultats des tests de fiabilité et de validité sont tout à fait satisfaisants $(\alpha=0,65$; rho $=0,81 ; A V E=0,57)$.

\section{Justification du choix des équations structurelles pour le test du modèle explicatif}

Bien que les données issues des plans expérimentaux soient par tradition analysées au moyen de la variance (ANOVA), nous avons fait le choix d'utiliser la méthode des équations structurelles (MES) pour traiter nos données. La MES présente de nombreux avantages. Tout d'abord, elle est très efficace pour le traitement de tout type de données dès lors que les variables sont mesurées avec des échelles numériques (Baron et Kenny, 1986: 1177). Ensuite, contrairement aux ANOVA, les équations structurelles peuvent traiter simultanément toutes les relations qui existent entre les variables d'un même modèle. Enfin, Iacobucci (2008) a montré que les équations structurelles constituent une méthode d'analyse supérieure aux ANOVA et aux régressions. Ces différentes raisons justifient notre choix de la MES pour le test du modèle.

\section{Résultats de la recherche}

Le premier modèle de fairness-based pricing (Figure 1) a permis de tester les effets individuels de chacune des variables indépendantes (équité perçue et transparence perçue) sur la réduction de l'injustice perçue et sur le CAP. Le second modèle (Figure 2) a porté sur le test des effets d'interaction entre une variable indépendante (l'équité perçue) et une variable modératrice (la transparence perçue). Les tableaux synoptiques des scores des paramètres estimés sont proposés dans les annexes 5 et 6 . Les des résultats commentés des différents tests sont présentés ci-après.

\section{Effets individuels de l'équité perçue et de la transparence perçue sur la réduction de l'injustice perçue et sur le CAP}

Afin de savoir si de façon individuelle, l'équité perçue du prix (EPP) et la transparence perçue de l'information (TPI) ont des effets sur la réduction 


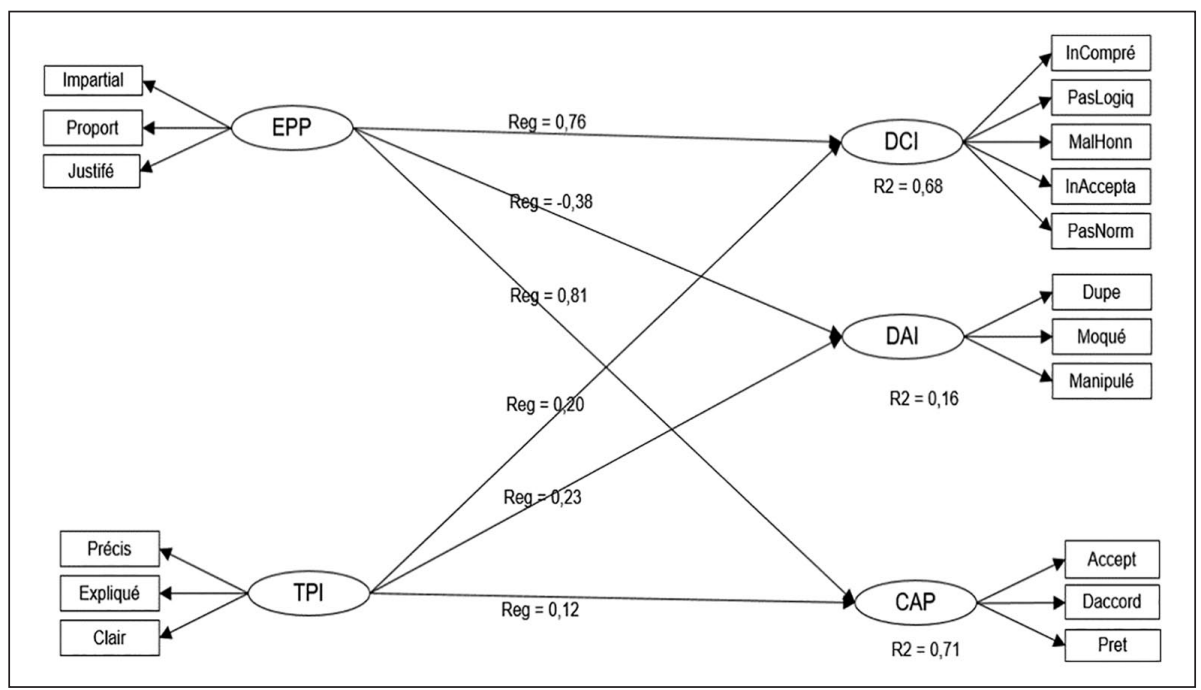

Figure 3. Modèle I avec les scores statistiques des effets testés

de l'injustice perçue et sur le CAP, nous avons procédé à l'analyse des effets individuels de chacune de ces deux variables. Les résultats sont présentés dans les paragraphes ci-après.

La figure 3 montre que sous les effets individuels de l'équité perçue du prix (EPP) et de la transparence perçue de l'information (TPI), les dimensions cognitive $\left(\mathrm{R}^{2}: 0,68\right)$ et affective $\left(\mathrm{R}^{2}: 0,16\right)$ de l'injustice perçue ont respectivement varié de $0,68 \%$ et de $0,16 \%$. Quant au consentement à payer $\left(\mathrm{R}^{2}: 0,71\right)$, sa variation est de $0,71 \%$. Il convient maintenant d'analyser le sens des relations et les scores dans chaque relation afin de pouvoir valider les hypothèses du premier modèle testé.

Effets de l'équité perçue du prix. Rappelons que la première condition de notre plan expérimental porte sur la mesure des effets de l'équité perçue sur l'injustice perçue et sur le CAP. Les scores des paramètres de cette condition expérimentale sont les suivants :

Le tableau 1 montre que l'équité perçue du prix a des effets positifs et significatifs sur la réduction de la dimension cognitive de l'injustice $(\beta: 0,76$; $\left.p<0,01 ; f^{2}: 1,70\right)$ et sur le $\operatorname{CAP}(\beta: 0,81 ; p<0,01$; $\left.f^{2}: 2,22\right)$. En revanche les effets sur la réduction de la dimension affective sont négatifs $(\beta:-0,38 ; p<$ 0,$\left.01 ; f^{2}: 0,10\right)$. Sur la base de ces éléments statistiques nous validons les hypothèses $\mathrm{H} 1$ et $\mathrm{H} 3$ et rejetons l'hypothèse $\mathrm{H} 2$.
Effets de la transparence perçue de l'information. Dans la deuxième condition expérimentale, nous avons testé les effets individuels de la transparence perçue sur l'injustice perçue et sur le CAP. L'objectif était de savoir si de façon individuelle, la transparence perçue permettait de réduire les perceptions d'injustice et de favoriser le CAP. Les scores statistiques obtenus sont montrés dans le tableau 2 :

Les scores du tableau 2 montrent que la transparence perçue (TPI) a des effets individuels positifs et significatifs sur la réduction des dimensions cognitive $(\beta: 0,20 ; P<0,01)$ et affective $(\beta: 0,23$; $P<0,01)$ de l'injustice perçue. Les statistiques montrent également que les effets de la transparence perçue sur le CAP sont positifs et significatifs $(\beta$ : $0,12 ; P>0,01)$. Ces statistiques nous permettent de valider toutes les hypothèses (H4, H5 et H6) sur les effets individuels de la transparence perçue de l'information avec un seuil très significatif de 0,01 .

\section{Effets d'interaction de l'équité perçue et de la transparence perçue sur la réduction de l'injustice perçue et sur le CAP}

Après avoir testé les hypothèses sur les effets individuels, nous avons cherché à savoir si l'équité perçue du prix (EPP) et la transparence perçue de l'information (TPI) avaient des effets d'interactions positifs et significatifs sur la réduction de l'injustice 
Tableau I. Scores des effets de l'équité perçue du prix (EPP).

\begin{tabular}{lllllll}
\hline $\begin{array}{l}\text { Variables } \\
\text { endogènes }\end{array}$ & $\begin{array}{l}\text { Variables } \\
\text { exogènes }\end{array}$ & $\beta$ & $\mathrm{ES}$ & $\mathrm{t}$ & $\mathrm{Pr}>|\mathrm{t}|$ & $\mathrm{f}^{2}$ \\
\hline Equité perçue & $\mathrm{DCl}$ & 0,76 & 0,03 & 21,70 & $0,00^{* * *}$ & $\mathrm{I}, 70$ \\
du prix (EPP) & DAl & $-0,38$ & 0,06 & $-6,68$ & 0,00 *** & 0,16 \\
& CAP & $0,8 \mathrm{I}$ & 0,03 & 24,72 & 0,00 *** & 2,21 \\
\hline
\end{tabular}

$\beta$ : régression; $E S$ : Erreur Standard; $t=$ test $T ; \operatorname{Pr}>F:$ Seuil de significativité; $f^{2}=$ Taille d'effet.

$\mathrm{DCl}$ : dimension cognitive de l'injustice perçue ; DAl : dimension affective de l'injustice perçue ;

CAP : consentement à payer.

Hypothèses

Validées

HI. L'équité perçue a un effet positif sur la réduction de la dimension cognitive de l'injustice

Oui*** perçue à l'égard de la TRM.

H2. L'équité perçue a un effet positif sur la réduction de la dimension affective de l'injustice

Non perçue à l'égard de la TRM.

H3. L'équité perçue a un effet positif sur le consentement à payer les prix découlant de la TRM.

Oui***

***: Hypothèse validée au seuil de $I \%=>P<0,0 I$

Tableau 2. Scores des effets de la transparence perçue de l'information (TPI).

\begin{tabular}{|c|c|c|c|c|c|c|}
\hline Variables endogènes & $\begin{array}{l}\text { Variables } \\
\text { exogènes }\end{array}$ & $\beta$ & ES & $\mathrm{t}$ & $\operatorname{Pr}>|t|$ & $f^{2}$ \\
\hline \multirow{3}{*}{$\begin{array}{l}\text { Transparence perçue } \\
\text { de l'information (TPI) }\end{array}$} & $\mathrm{DCl}$ & 0,20 & 0,03 & 5,66 & $0,00 * * *$ & 0,12 \\
\hline & DAI & 0,23 & 0,06 & 4,14 & $0,00 * * *$ & 0,06 \\
\hline & CAP & 0,12 & 0,03 & 3,58 & $0,00 * * *$ & 0,05 \\
\hline
\end{tabular}

$\beta$ : régression; ES: Erreur Standard; $t=$ test $T ; \operatorname{Pr}>F$ : Seuil de significativité; $f^{2}=$ Taille d'effet.

$\mathrm{DCl}$ : dimension cognitive de l'injustice perçue; DAl: dimension affective de l'injustice perçue;

CAP: consentement à payer.

Hypothèses

Validées

H4. La transparence perçue $a$ un effet positif sur la réduction de la dimension cognitive de l'injustice perçue à l'égard de la TRM

H5. La transparence perçue $a$ un effet positif sur la réduction de la dimension affective de l'injustice Oui*** perçue à l'égard de la TRM

H6. La transparence perçue a un effet positif sur le consentement à payer les prix découlant de la TRM.

Oui***

***: Hypothèse validée au seuil de $I \%=>P<0,01$

perçue et sur le CAP. Rappelons que dans nos hypothèses sur les interactions (modèle 2), l'équité perçue constitue la variable indépendante et la transparence perçue est utilisée en tant que variable modératrice. Trois effets d'interactions ont été testés au moyen des régressions par les moindres carrés partiels (PLS) puis au moyen du test de Chow. 


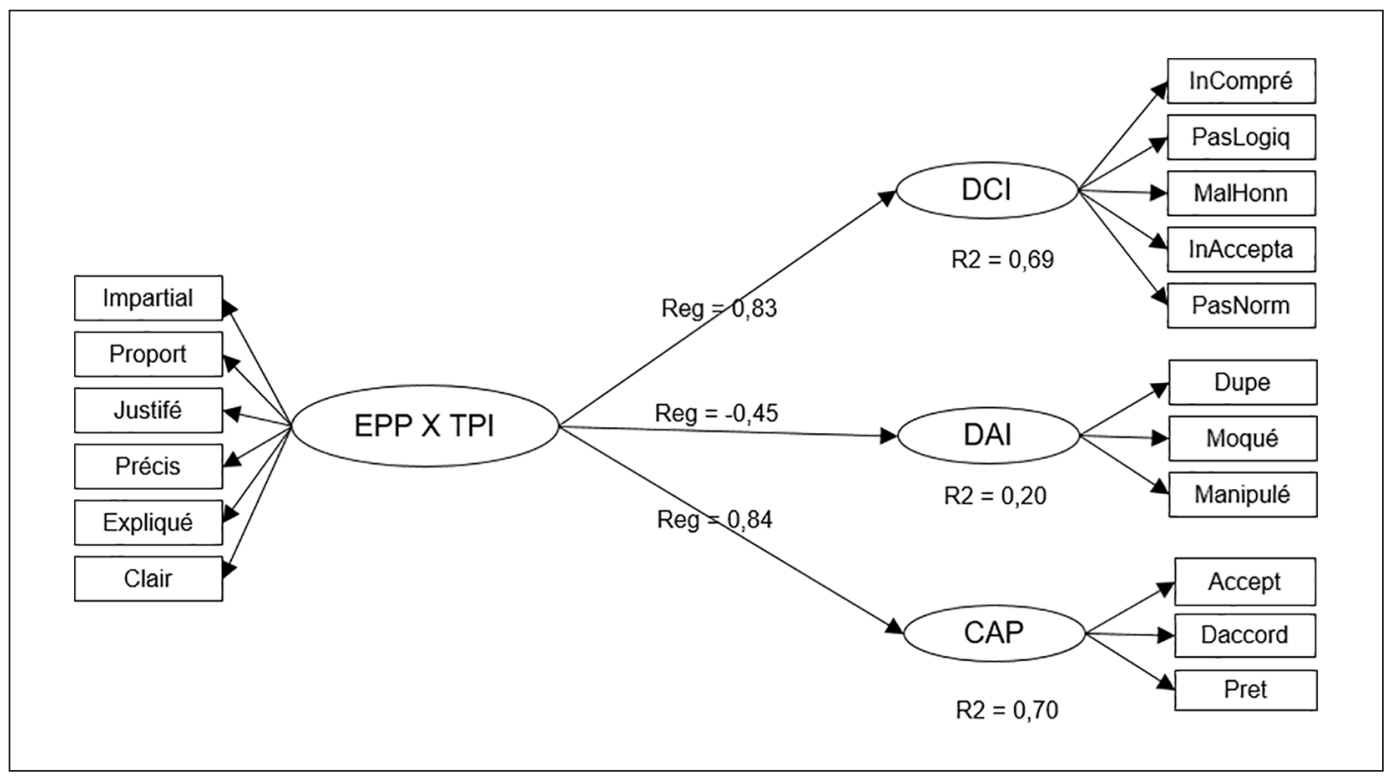

Figure 4. Modèle 2 avec les scores des effets d'interaction.

Tableau 3. Effets d'interaction de l'équité perçue (EPP) et de la transparence perçue (TPI).

\begin{tabular}{llrlrrr}
\hline $\begin{array}{l}\text { Effets } \\
\text { d'interaction }\end{array}$ & $\begin{array}{l}\text { Variables } \\
\text { exogènes }\end{array}$ & \multicolumn{1}{l}{ B } & ES & $\mathrm{t}$ & $\operatorname{Pr}>|\mathrm{t}|$ & $\mathrm{f}^{2}$ \\
\hline EPP X TPI & DCl & 0,83 & 0,03 & 24,66 & $0,00^{* * * *}$ & 2,19 \\
& DAI & $-0,45$ & 0,05 & $-8,30$ & $0,00^{* * *}$ & 0,25 \\
& CAP & 0,84 & 0,03 & 25,73 & $0,00^{* * *}$ & 2,38
\end{tabular}

$\beta$ : régression; ES: Erreur Standard; $t=$ test $T ; \operatorname{Pr}>F$ : Seuil de significativité; $f^{2}=$ Taille d'effet.

$\mathrm{DCl}$ : dimension cognitive de l'injustice perçue; DAI: dimension affective de l'injustice perçue.

CAP: consentement à payer; EPP: équité perçue du prix; TPI: transparence perçue de l'information.

Test des interactions par la méthode des moindres carrés partiels333. Les résultats des tests d'interactions par la méthode des moindres carrés partiels sont présentés sur la figure 4 et dans le tableau 3 ci-après.

La figure 4 montre que l'interaction entre l'équité perçue (EPP) et la transparence perçue (TPI) a des effets sur les dimensions cognitive $\left(R^{2}: 0,69\right)$ et affective $\left(\mathrm{R}^{2}: 0,20\right)$ de l'injustice perçue à l'égard de la TRM. Cette interaction a aussi des effets sur le CAP $\left(\mathrm{R}^{2}: 0,70\right)$. Le sens des relations et les scores statistiques ci-après, permettront de valider ou de rejeter les hypothèses sur les effets d'interaction du modèle.

\section{Comparaison du modèle $I$ et du modèle 2 avec le test de Chow}

Les coefficients de régression présentés ci-dessus montrent des différences entre les effets de l'équité perçue dans le modèle 1 (modèle sans interactions avec la transparence) et les effets de cette même variable indépendante dans le modèle 2 (modèle d'interactions), indiquées dans le tableau 4.

Afin de savoir si les différences observées (tableau 4) sont significatives, un test de Chow avec des analyses multigroupes (Gavard-Perret et al., 2012: 323; Addinsoft, 2019) a été réalisé. Les résultats sont les suivants : 
Tableau 4. Comparaison entre le modèle I et le modèle 2.

\begin{tabular}{lll}
\hline $\begin{array}{l}\text { Variables } \\
\text { exogènes }\end{array}$ & $\begin{array}{l}\text { Modèle I } \\
\text { Effets de l'équité perçue sans modé- } \\
\text { ration de la transparence perçue }\end{array}$ & $\begin{array}{l}\text { Modèle 2 } \\
\text { Effets de l'équité perçue avec modé- } \\
\text { ration de la transparence perçue }\end{array}$ \\
\hline $\mathrm{DCl}$ & 0,76 & 0,83 \\
$\mathrm{DAI}$ & $-0,38$ & $-0,45$ \\
$\mathrm{CAP}$ & 0,81 & 0,84 \\
\hline
\end{tabular}

DCl: dimension cognitive de l'injustice perçue; DAl: dimension affective de l'injustice perçue;

CAP: consentement à payer.

Tableau 5. Résultats du test de Chow.

\begin{tabular}{lllllll}
\hline $\begin{array}{l}\text { Variables } \\
\text { exogènes }\end{array}$ & $\begin{array}{l}\beta \\
\text { (Différence) }\end{array}$ & $\begin{array}{l}\mathrm{t} \\
\text { (observé) }\end{array}$ & $\begin{array}{l}\mathrm{t} \\
\text { (critique) }\end{array}$ & DDL & P-value & Significatif \\
\hline $\mathrm{DCl}$ & 0,23 & 2,29 & 1,96 & 558 & $0,02^{* *}$ & Oui \\
DAI & 0,25 & 1,33 & 1,96 & 558 & 0,18 & Non \\
CAP & 0,97 & 4,35 & 1,96 & 558 & $0,01^{* *}$ & Oui \\
\hline
\end{tabular}

DCl: dimension cognitive de l'injustice perçue; DAl: dimension affective de l'injustice perçue;

CAP: consentement à payer; $* *=\mathrm{P}<0,05$.

Hypothèses

Validée

H7. L'interaction entre l'équité perçue et la transparence perçue a des effets positifs sur la Oui** réduction de la dimension cognitive de l'injustice perçue à l'égard de la TRM.

H8. L'interaction entre l'équité perçue et la transparence perçue a des effets positifs la réduction

Non de la dimension affective de l'injustice perçue à l'égard de la TRM.

H9. L'interaction entre l'équité perçue et la transparence perçue a des effets positifs sur le consentement à payer.

**: Hypothèse validée au seuil de $I \%=>P<0,05$

Le test de Chow (tableau 5) montre des différences positives et significatives entre le modèle sans interaction (modèle 1) et le modèle avec interactions (modèle 2 ) au niveau de la dimension cognitive de l'injustice perçue $(\beta: 0,23$; $P<0,01)$ et au niveau du CAP $(\beta: 0,97 ; P<0,01)$. En revanche, la différence n'est pas significative au niveau de la dimension affective de l'injustice perçue $(\beta: 0,25 ; P<0,18)$. Les scores du test de Chow permettent de valider les hypothèses $\mathrm{H} 7$ et $\mathrm{H} 9$ et de rejeter l'hypothèse H8.

\section{Conclusion générale: apports, limites et voies de recherche}

Cet article a présenté les résultats d'une recherche sur la perception et le consentement à payer des prix basés sur la TRM. Les apports de cette recherche sont multiples tant au niveau théorique que managérial.

\section{Les apports théoriques}

Sur le plan théorique, quatre principales contributions sont issues de cette recherche. La première porte sur la validation empirique de deux modèles explicatifs de réduction de l'injustice perçue et du consentement à payer les prix découlant de la TRM. En effet, comme précisé plus haut, malgré une littérature foisonnante, peu de modèles empiriques sur les stratégies de réduction de l'injustice perçue et sur le CAP ont été testés dans le contexte de la TRM. Ensuite, la littérature est totalement muette en ce qui concerne les effets 
d'interactions entre l'équité perçue et la transparence perçue dans le contexte de la TRM. Les modèles existants sont généralement exploratoires et les postulats sur les leviers de réduction de l'injustice perçue et sur le CAP ont été peu mis à l'épreuve des faits. Notre recherche a contribué à combler cette insuffisance de la littérature en validant deux modèles de «fairness-based pricing» ou tarification basée sur la justice. Le premier modèle suggère que les variables perceptuelles, singulièrement l'équité perçue et la transparence perçue, ont des effets individuels positifs directs sur la réduction de l'injustice perçue et sur le consentement à payer dans le contexte de la TRM. Plus précisément, la validation des hypothèses $\mathrm{H} 1$ et $\mathrm{H} 3$ montre que l'équité perçue du prix (EPP) a des effets positifs directs sur la réduction de la dimension cognitive de l'injustice perçue à l'égard de la TRM et sur le CAP. Ces résultats renforcent les théories de l'équité perçue (Oliver et Swan, 1989; Xia et Monroe, 2010) et de l'intérêt mutuel (Kahneman et al., 1989) qui postulent que la présence de l'équité dans une politique de prix permet d'atténuer la dimension cognitive de l'injustice perçue. A contrario, le rejet de $\mathrm{H} 2$ suggère que l'équité perçue du prix n'a pas d'effets significatifs sur la dimension affective de l'injustice perçue. Le rejet de cette hypothèse nous enseigne que la présence de l'équité ne suffit pas toujours pour réduire les affects négatifs qu'un consommateur peut éprouver dans le contexte de la TRM. Ce résultat renforce les modèles qui postulent que dans le contexte de la TRM, quel que soit le prix payé par le client (inférieur ou supérieur au prix espéré), les risques d'injustice perçue peuvent être présents (Camus et al., 2014). En effet, dans le cas d'un prix avantageux, malgré l'équité perçue, le prix basé sur le RM peut induire des émotions négatives (colère, dégout, culpabilité, etc.) à cause du caractère relativement opaque et discriminatoire de la TRM (Granados, et al., 2018). Concernant les effets de la transparence perçue, la validation de toutes les hypothèses $(\mathrm{H} 4, \mathrm{H} 5$ et $\mathrm{H} 6)$ relatives aux effets individuels de ce facteur montre l'importance capitale de l'information dans le contexte de la TRM. Ces résultats nous informent que les consommateurs ont besoin de savoir pourquoi dans certains secteurs, ils paient des prix différents pour une même catégorie de produit (par exemple, un billet de train sur un même trajet et sur le même créneau horaire). $\mathrm{Si}$ le consommateur a des informations claires et fiables sur les raisons qui justifient une différence de prix, il sera plus enclin à accepter la TRM et à payer les prix découlant de cette pratique.

La deuxième contribution théorique concerne la comparaison des performances des deux modèles testés: l'un avec deux variables indépendantes (équité perçue et transparence perçue), l'autre avec une variable indépendante (équité perçue) et une variable modératrice (transparence perçue). Le test de Chow a permis de montrer que l'interaction entre l'équité perçue et la transparence perçue a des effets significatifs en matière de réduction de la dimension cognitive de l'injustice perçue et en matière du consentement à payer. La validation des hypothèses $\mathrm{H} 7$ et $\mathrm{H} 9$ nous informe que pour réduire l'injustice perçue et favoriser le consentement payer, il est nécessaire de mobiliser simultanément des leviers d'équité (par exemple, le prix en fonction de la taille d'une chambre) et de transparence (information claire et fiable, etc.). Toutefois, le rejet de l'hypothèse $\mathrm{H} 8$ nous renseigne que le recours à la transparence (en tant que modérateur) ne suffit pas toujours pour réduire les affects négatifs dans le contexte de la TRM. D'autres variables (par exemple, le respect de l'éthique par l'entreprise, la responsabilité sociétale de l'entreprise, etc.) feraient aussi partie des critères pris en compte par les consommateurs pour évaluer la justice des prix. Au final, nous concluons que les deux modèles testés sont à peu près équivalents en matière de performance pour réduire l'injustice perçue et pour renforcer le CAP. Les deux modèles ont en commun de rejeter les hypothèses relatives à la dimension affective de l'injustice perçue, sauf dans le cas de l'effet individuel de la transparence perçue. En revanche, ils valident de façon concurrente toutes les hypothèses sur la réduction de la dimension cognitive de l'injustice et sur le renforcement du CAP. Par ailleurs, la comparaison des performances de l'équité perçue avec celles de la transparence perçue montre que l'équité perçue a des effets positifs sur la dimension cognitive et sur le CAP mais pas sur la dimension affective, que ce soit dans le premier ou dans le second modèle. Quant à la transparence perçue, ses effets sont tous positifs dans les deux modèles, sauf sur la réduction de la 
dimension affective du deuxième modèle. Ces résultats nous autorisent à tirer une deuxième conclusion qui suggère que la transparence perçue (que ce soit dans son statut de variable indépendante ou dans son rôle de variable modératrice) apparait comme un facteur fondamental dans la réduction des dimensions cognitives et affectives de l'injustice perçue et dans le renforcement du CAP. L'équité perçue, quant à elle, est très efficace dans la réduction de la dimension cognitive de l'injustice perçue et pour favoriser le CAP. Mais elle est insuffisante pour réduire la dimension affective de l'injustice perçue. Notre recherche a donc permis de mettre en évidence les limites des stratégies tarifaires focalisées sur l'équité et de montrer l'importance de la transparence perçue. Ces conclusions corroborent les théories selon lesquelles la dimension affective de l'injustice perçue est généralement difficile à réduire lorsque les stratégies de la TRM se limitent à l'amélioration de l'équité sans prendre en compte d'autres facteurs. En effet, au-delà de l'équité et de la transparence, d'autres variables seraient également importantes pour le consommateur dans le contexte de la TRM. Il s'agit par exemple, des facteurs relatifs au respect par l'entreprise des normes sociales (Maxwell, 2008; Meatchi et Camus, 2018a), de l'éthique (Goldeman et Cropanzano, 2014; Pez et al., 2017) ou encore la responsabilité sociétale de l'entreprise (Koschate-Fischer et al., 2016; Thiery, 2005). L'intégration de ces variables dans une stratégie de TRM permettrait peut-être de mieux de réduire les dimensions affectives de l'injustice perçue et de renforcer le CAP.

La troisième contribution théorique est relative à la mobilisation de la théorie de la justice des prix (Xia et al., 2004) comme principal cadre d'analyse dans cette recherche. Il s'agit d'une contribution importante car peu de recherches antérieures s'étaient appuyées sur ce cadre théorique. La plupart des modèles existants ont utilisé soient le principe de l'intérêt mutuel ou dual entitlement (Kahneman et al., 1986), soit les théories de la justice organisationnelle (Greenberg, 1987) comme socle d'analyse de la perception des prix. Or ces théories sont parcellaires et ne permettent pas d'appréhender de façon intégrative les problématiques liées à la justice et au consentement à payer des prix découlant de la TRM (Xia et al., 2004). En mobilisant la théorie de la justice des prix, notre recherche apporte une vision plus large sur les stratégies de réduction de l'injustice perçue et du renforcement du CAP. La théorie de la justice des prix a permis de prendre simultanément en compte les dimensions cognitives et affectives de l'injustice perçue mais aussi de mobiliser l'équité et la transparence dans un même modèle explicatif. Cette approche intégrative est peu présente dans les modèles fondés sur les théories de la justice organisationnelle (Greenberg, 1987) ou sur le principe de l'intérêt mutuel (Kahneman et al., 1986) qui sont très centrés sur les variables cognitives au détriment des variables affectives.

Enfin, la quatrième et dernière contribution théorique concerne la clarification conceptuelle, la mesure en deux dimensions et la validation prédictive du concept de l'injustice perçue à l'égard de la TRM. Nous avons mobilisé ce concept après l'avoir défini et mesuré en deux dimensions (cognitive et affective). Ensuite, nous avons testé sa validité prédictive en l'intégrant dans notre modèle explicatif. Ces différentes validités psychométriques permettent de conférer à l'injustice perçue un statut théorique clair permettant de la différencier des concepts proches ou opposés tels que la justice perçue; ce qui n'avait pas encore été fait dans la littérature sur la perception des prix en général et dans les modèles sur la perception de la TRM, en particulier.

\section{Les apports managériaux}

Sur le plan managérial, les implications de cette recherche sont également nombreuses. Les entreprises hôtelières sont généralement partagées entre les avantages du revenue management et les risques d'injustice perçue liés à cette pratique. $\mathrm{Si}$ l'intérêt de la TRM n'est plus à démontrer, la préoccupation des professionnels (en particulier de la petite et moyenne hôtellerie) est de savoir comment faire une tarification par le revenue management (TRM) en limitant les risques d' "effet boomerang» de cette pratique. Notre recherche a tenté de répondre à cette problématique en proposant une modélisation de TRM basée sur l'équité du prix et sur la transparence perçue de l'information. Nous qualifions cette modélisation de «fairness-based 
pricing» (tarification basée sur la justice). Il ne s'agit pas d'une modélisation révolutionnaire mais d'une approche incrémentale qui invite les professionnels à prendre en considération les variables perceptuelles (équité perçue, transparence perçue, etc.) dans leurs pratiques du revenue management. Dans cette optique et afin de répondre aux attentes des consommateurs en matière d'équité et de transparence, les hypothèses validées dans cette recherche suggèrent aux managers de compléter les modèles traditionnels basés sur les historiques de vente et sur les approches stochastiques (Belobaba, 1989; Koch et al., 2017) en y intégrant des leviers d'équité et de transparence. L'intégration de l'équité dans les modèles de TRM peut passer par des techniques d'enrichissement de la valeur de l'offre (Rivière et Bourliataux-Lajoinie, 2017; Xia et Monroe, 2010). En d'autres termes, pour mettre en œuvre une politique de pricing basée sur l'équité, l'entreprise doit enrichir la valeur de l'offre lorsqu'elle veut afficher des prix plus élevés. Inversement, si elle sent le besoin d'afficher des prix plus bas afin de stimuler la demande, elle devra revoir certains attributs de l'offre afin que les clients ayant déjà payé les prix les plus élevés n'aient pas l'impression d'avoir été lésés. Les clients attendent que la différence de prix soit justifiée par une différence de valeur de l'offre et pas uniquement selon la date de réservation (modèle calendaire) ou selon la pression de la demande (modèle de courbesseuils). De même, une stratégie de différenciation fondée sur les attributs hédonistes et sensoriels (par exemple, la vue offerte par une chambre, la qualité de la décoration d'une chambre, etc.) ou instrumentaux (accès au $\mathrm{Wi}-\mathrm{Fi}$, minibar, etc.) peut être utilisée comme ingrédient d'une tarification équitable. La gestion des prix à partir de l'équité et de la valeur de l'offre permet à la fois de répondre aux principes de l'équité interne et externe (Maxwell, 2008). Cependant, une politique d'équité serait vaine si l'entreprise n'est pas transparente sur sa politique de pricing. La mise à disposition des clients d'une information claire et précise sur la politique tarifaire est nécessaire afin de réduire les réactions cognitives et affectives négatives à l'égard de la TRM. Les recherches antérieures (par exemple, Ayadi et al., 2017) ont montré que la clarté, la précision, la cohérence et la fiabilité de l'information sur les prix sont des critères essentiels qui permettent au consommateur de prendre sa décision lors d'un processus d'achat. La transparence de l'information constitue l'un des indicateurs les plus utilisés par les consommateurs pour évaluer la justice (procédurale et distributive) dans le contexte des prix. Les hypothèses validées dans cette recherche confirment les postulats proposés dans les études antérieures et montrent que la mise à disposition d'une information transparente est l'un des leviers clés permettant de réduire les perceptions d'injustice et de favoriser le consentement à payer. Les entreprises de services comme la SNCF ont pris conscience des enjeux de la transparence de l'information sur les prix. La SNCF a en effet mis en place, depuis 2017, une politique de transparence dénommée programme «Information First». Ce programme a été engagé pour répondre à l'insatisfaction très forte exprimée par les clients sur l'information fournie par la société ferroviaire. Le vrai changement derrière ce programme, c'est la priorité donnée à l'information client.

\section{Limites de la recherche}

Concernant les limites, nous sommes conscients que notre recherche comporte des faiblesses qu'il convient d'évoquer avant d'envisager de nouvelles voies de recherche. Premièrement, le modèle global de fairness-based pricing que nous avons testé s'est concentré sur deux variables de la justice, à savoir l'équité perçue et la transparence perçue. D'autres dimensions de la justice (par exemple, l'éthique perçue, la valeur perçue de l'offre, etc.) n'ont pas été prises en compte dans le modèle. L'absence de ces variables serait peut-être à l'origine du rejet de l'hypothèse $\mathrm{H} 2$ dans le premier modèle et de l'hypothèse $\mathrm{H} 8$ dans le second modèle. Malgré la présence de l'équité et de la transparence, les consommateurs peuvent ressentir de l'injustice s'ils estiment que l'entreprise ne respecte pas les principes d'éthique (Ayadi et al., 2017) ou que le niveau d'un prix est en décalage avec la valeur de l'offre correspondante (Xia et Monroe, 2010). Deuxièmement, notre modèle n'intègre pas de variables de contingence ni de variables relatives 
aux caractéristiques individuelles (revenus, sensibilité au prix, familiarité avec le RM, etc.) et sociodémographiques (âges, sexe, profession, etc.). Or, les recherches antérieures (ex.: Heo et Lee, 2011) ont montré que les caractéristiques individuelles des consommateurs et certains facteurs de contingence (par exemple, l'état de la concurrence, la nationalité et la culture du client, etc.) jouent un rôle important dans la perception de la TRM. Une intégration des variables situationnelles aurait été intéressante afin de savoir si ces variables ont un impact sur la perception du revenue management et sur le consentement à payer les prix basé sur cette technique. Enfin, des limites méthodologiques sont aussi à prendre en considération. Les scénarios proposés dans le plan expérimental comportent probablement des biais. Même si nos scénarios ont été construits avec la plus grande rigueur et avec l'aide des professionnels, ces outils restent néanmoins des schémas théoriques dont la mise en pratique dans la «vraie vie» pourrait ne pas donner les mêmes résultats que ceux de cette recherche. Leur test en condition réelle dans les hôtels sera nécessaire afin de prouver leur robustesse. Aussi, les échantillons utilisés dans cette recherche ne sont pas parfaitement représentatifs. Certaines catégories socioprofessionnelles telles que les retraités (4\%) sont peu représentées.

\section{Perspectives et voies de recherches futures}

Nous ne saurons terminer cet article sans proposer des voies de recherche. Premièrement, nous suggérons que l'échelle de mesure de l'injustice perçue soit à nouveau mise à l'épreuve des faits dans d'autres contextes afin de prouver sa performance et surtout sa validité externe. Le déploiement de cet instrument sur d'autres problématiques de la TRM et dans d'autres secteurs d'activité (par exemple, le transport ferroviaire, la restauration, les parcs à thème, etc.) sera un moyen de confirmer sa fiabilité et sa validité interne et externe. Concernant la réduction de l'injustice perçue et l'amélioration du consentement à payer, nous suggérons que d'autres leviers stratégiques de réduction de l'injustice perçue à l'égard de la TRM soient étudiés. Il peut s'agir par exemple des leviers relatifs à l'éthique, à la justice procédurale et interactionnelle qui n'ont pas été abordés dans cette recherche. Des leviers basés sur la valeur perçue de l'offre (Rivière et Mencarelli, 2012) sont aussi des voies de recherche à explorer. Puis, des expérimentations en condition réelle de notre modèle (fairness-based pricing, FBP) doivent être réalisées dans les futures recherches. Ces nouveaux tests permettraient de prouver l'efficacité opérationnelle des deux modèles proposés. Il s'agira par exemple de collaborer avec des hôtels qui accepteraient de s'engager dans une démarche de pricing basée sur l'équité et sur la transparence. Il serait ainsi possible de mesurer les réactions des clients «réels» face à un modèle de TRM basé sur l'équité et la transparence mobilisées en tant que variables indépendantes et un modèle basé sur l'équité prise comme variable indépendante et la transparence utilisée comme variable modératrice. Notre recherche ouvre donc de nouvelles voies d'investigation concernant les stratégies de réduction de l'injustice perçue à l'égard des politiques de TRM et les leviers d'actions mobilisables pour favoriser le consentement à payer. Par ailleurs, des tests de médiation (par exemple, le rôle médiateur de l'injustice perçue dans la relation entre l'équité perçue et le CAP) et d'autres tests de modération (par exemple les effets modérateurs des variables individuels des consommateurs) sont aussi des voies à explorer dans les futures recherches.

\section{ORCID iDs}

Sourou MEATCHI (iD https://orcid.org/0000-0003-0125 $-6393$

Sandra CAMUS (iD https://orcid.org/0000-0002-4710 $-9551$

\section{Références bibliographiques}

Abrate G, Nicolau JL et Viglia G (2019) The impact of dynamic price variability on revenue maximization. Tourism Management 74: 224-233.

Adams JS (1965) Inequity in social exchange. In: Advances in Experimental Social PsychologyAcademic Press, Vol. 2, 267-299.

Adamy J (2000) E-Tailer price tailoring may be wave of future," Chicago Tribune, (September 25), 4. 
Addinsoft (2019) XLSTAT statistical and data analysis solution. Long Island NY, USA. https://www.xlstat.com

Ayadi N, Paraschiv C et Rousset X (2017) Tarification dynamique en ligne et éthicalité perçue par le consommateur: synthèse et voies de recherche. Recherche et Applications en Marketing 32(3), 53-75.

Baron RM et Kenny DA (1986) The moderatormediator variable distinction in social psychological research: conceptual, strategic, and statistical considerations. Journal of Personality and Social Psychology 51(6): 1173.

Bearden WO, Carlson JP et Hardesty DM (2003) Using invoice price information to frame advertised offers. Journal of Business Research 56(5): 355-366.

Belobaba P (1989) Application of a probabilistic decision model to airline seat inventory control. Operations Research 37(2): 183-197.

Bolton LE, Warlop L et Alba JW (2003) Consumer perceptions of price (un) fairness. Journal of Consumer Research 29(4): 474-491.

Brown-Liburd H, Cohen J et Zamora VL (2018) CSR disclosure items used as fairness heuristics in the investment decision. Journal of Business Ethics 152(1): 275-289.

Buckhiester B (2011) Revenue management as a multidisciplinary business process: part two. The Journal of Hospitality Financial Management 19(2): 97-113.

Campbell MC (2007) "Says who?!" How the source of price information and affect influence perceived price (un) fairness. Journal of Marketing Research 44(2): 261-271.

Camus S, Hikkerova L et Sahut JM (2014) L'injustice perçue à l'égard du yield management: antécédents et stratégies de réduction. Décisions Marketing (73): 45-63.

Capiez A (2003) Yield management. Optimisation du revenu dans les services. Paris: Lavoisier.

Choi S et Mattila AS (2005) Impact of information on customer fairness perceptions of hotel revenue management. Cornell Hotel and Restaurant Administration Quarterly 46(4): 444-51.

Churchill GA Jr (1979) A paradigm for developing better measures of marketing constructs. Journal of Marketing Research 16(1): 64-73.

Colquitt JA, Long DM, Rodell JB et Halvorsen-Ganepola MD (2015) Adding the "in" to justice: a qualitative and quantitative investigation of the differential effects of justice rule adherence and violation. Journal of Applied Psychology 100(2): 278.

Deutsch M (1975) Equity, equality, and need: what determines which value will be used as the basis of distributive justice? Journal of Social Issues 31(3): 137-149.
Dodds WB, Monroe KB et Grewal D (1991) Effects of price, brand, and store information on buyers' product evaluations. Journal of Marketing Research. 28(3): 307-319.

Domingo-Carrillo MÁ, Chávez-Miranda E et EscobarPérez B (2019) Scientific production on revenue management in tourism on Web of Science and SCOPUS. Current Issues in Tourism 23(7): 1-26.

Ferguson JL, Ellen, PS et Bearden, WO (2014) Procedural and distributive fairness: determinants of overall price fairness. Journal of Business Ethics 121(2): 217-231.

Fornell C and Larcker DF (1981) Structural equation models with unobservable variables and measurement error: Algebra and statistics. Journal of Marketing Research: 382-388.

Gavard-Perret ML, Gotteland D, Haon C et Jolibert A (2012) Méthodologie de la recherche en sciences de gestion. Réussir son mémoire ou sa thèse, 2. Paris: Pearson.

Goldman B et Cropanzano R (2015) "Justice" and "fairness" are not the same thing. Journal of Organizational Behavior 36(2): 313-318.

Granados N, Han K et Zhang D (2018) Demand and revenue impacts of an opaque channel: evidence from the airline industry. Production and Operations Management 27(11), 2010-2024.

Greenberg J (1987) A taxonomy of organizational justice theories. Academy of Management Review 12(1): 9-22.

Grewal D, Monroe KB et Krishnan R (1998) The effects of price-comparison advertising on buyers' perceptions of acquisition value, transaction value, and behavioral intentions. Journal of Marketing 62(2): 46-59.

Heyman JE et Mellers BA (2008) Perceptions of fair pricing. In: Haugtvedt CP, Herr MP et Kardes FR (éds), Handbook of Consumer Psychology. Mawah NJ: Lawrence Erlbaum Associates, 683-697.

Heo CY et Lee S (2011) Influences of consumer characteristics on fairness perceptions of revenue management pricing in the hotel industry. International Journal of HospitalityManagement 30(2): 243-251.

Iacobucci D (2008) Mediation analysis. Thousand Oaks CA: SAGE.

Inman JJ et Nikolova H (2017) Shopper-facing retail technology: a retailer adoption decision framework incorporating shopper attitudes and privacy concerns. Journal of Retailing 93(1): 7-28.

Jarvis CB, MacKenzie SB et Podsakoff PM (2003) A critical review of construct indicators and measurement model misspecification in marketing and consumer research. Journal of Consumer Research 30(2): 199-218. 
Kahneman D, Knetsch JL et Thaler RH (1986) Fairness as a constraint on profit seeking: entitlements in the market. The American Economic Review 76(4): 728-741.

Kimes SE et Wirtz J (2002), Perceived fairness of revenue management in the US golf industry. Journal of Revenue and Pricing 1(4): 332-344.

Kimes SE et Wirtz J (2015) Revenue management: advanced strategies and tools to enhance firm profitability. Foundations and Trends ${ }^{\circledR}$ in Marketing 8(1): 1-68.

Kimes S et Wirtz J (2016) Revenue management in restaurants: unbundling pricing for reservations from the core service.Cornell Hospitality Report, 16(8): 3-12.

Kimes SE (1994) Perceived fairness of yield management: applying yield-management principles to rate structures is complicated by what consumers perceive as unfair practices. Cornell Hotel and Restaurant Administration Quarterly 35(1): 22-29.

Koch S, Gönsch J et Steinhardt C (2017) Dynamic programming decomposition for choice-based revenue management with flexible products. Transportation Science 51(4): 1046.

Koschate-Fischer N, Huber I V et Hoyer W D (2016). When will price increases associated with company donations to charity be perceived as fair? Journal of the Academy of Marketing Science 44(5): 608-626.

Lavorata L, Nillès JJ et Pontier S (2005) La méthode des scénarios: une méthode qualitative innovante pour le marketing. Application au comportement éthique du vendeur en B to B. Décisions Marketing 37: 67-75.

Le Gall-Ely ML (2009) Définition, mesure et déterminants $d u$ consentement à payer du consommateur: synthèse critique et voies de recherche. Recherche et Applications en Marketing 24(2): 91-11.

Li X, Li KJ et Wang X (2019) Transparency of behaviorbased pricing. Journal of Marketing Research 57(1): 78-99.

Lind EA (2001) Fairness heuristic theory: justice judgments as pivotal cognitions in organizational relations. In: Greenberg J et Cropanzano R (éds) Advances in Organization Justice. Redwood City CA: Stanford University Press, 56-88.

Lind EA (1992) The fairness heuristic: rationality and "relationality" in procedural evaluations. In: Fourth International Conference of the Society for the Advancement of Socio-Economics, Irvine, CA.

Lu Z, Bolton LE, Ng S et Chen HA (2019) The Price of Power: How Firm's Market Power Affects Perceived Fairness of Price Increases. Journal of Retailing Disponible en ligne: https://doi.org/10.1016/j.jretai.2019.09.004

Maxwell S (2008) The Price is Wrong: Understanding What Makes a Price Seem Fair and The True Cost of Unfair Pricing. Hoboken NJ: John Wiley and Sons.

Maxwell S (2002) Rule-based price fairness and its effect on willingness to purchase. Journal of Economic Psychology 23(2): 191-212.

Meatchi S et Camus S (2018a) L'injustice perçue à l'égard de la tarification par le revenue management: une analyse de ses manifestations affectives. Management \& Avenir (1(99): 143-164.

Meatchi S et Camus S (2018b) Proposition d'une échelle de mesure du concept de l'injustice perçue à l'égard de la tarification par le revenue management: une version exploratoire. 34ème Conférence de l'Association Française du Marketing. Strasbourg, 2018.

Miao L et Mattila AS (2007) How and how much to reveal? The effects of price transparency on consumers' price perceptions. Journal of Hospitality et Tourism Research 31(4): 530.

Morwitz VG, Greenleaf, EA et Johnson EJ (1998) Divide and prosper: consumers' reactions to partitioned prices. Journal of Marketing Research 35(4): 453-463.

Noone BM et Mattila AS (2009) Hotel revenue management and the internet: the effect of price presentation strategies on customers' willingness to book. International Journal of Hospitality Management 28(2): 272-279.

Noone BM (2016) Pricing for hotel revenue management: evolution in an era of price transparency. Journal of Revenue and Pricing Management 15(34): $264-269$

Oliver RL et Swan JE (1989a) Equity and disconfirmation perceptions as influences on merchant and product satisfaction. Journal of Consumer Research 16(3): 372-383

Oliver RL et Swan JE (1989b) Consumer perceptions of interpersonal equity and satisfaction in transactions: a field survey approach. Journal of Marketing 53(2): 21-35.

Pez V, Butori R et Mimouni-Chaabane A (2017) Le côté sombre de la pression exercée sur les consommateurs par les programmes de fidélité: enjeux éthiques et pratiques. Recherche et Applications en Marketing 32(3): 76-89.

Rivière A et Mencarelli R (2012) Vers une clarification théorique de la notion de valeur perçue en marketing. Recherche et Applications en Marketing 27(3): 97-123.

Rivière A et Bourliataux-Lajoinie S (2017) Les effets des m-services touristiques sur la proposition de valeur d'une ville. Decisions Marketing 85: 101-119. Disponible en ligne:http://10.7193/DM.085.101.119. 
Rossiter JR (2002). The C-OAR-SE procedure for scale development in marketing. International Journal of Research in Marketing 19(4): 305-335.

Rossiter JR (2011). Marketing measurement revolution: The C-OAR-SE method and why it must replace psychometrics. European Journal of Marketing 45: 1589-1600.

Rupp DE, Shapiro DL, Folger R, Skarlicki DP et Shao R (2017) A critical analysis of the conceptualization and measurement of organizational justice: is it time for reassessment? Academy of Management Annals 11(2): 919-959.

Sahut JM, Hikkerova L et Pupion PC (2016) Perceived unfairness of prices resulting from yield management practices in hotels. Journal of Business Research 69(11): 4901-4906.

Tanford S, Baloglu S et Erdem M (2012) Travel packaging on the Internet: the impact of pricing information and perceived value on consumer choice. Journal of Travel Research 51(1): 68-80.

Tanford S, Choi C et Joe SJ (2018) How pricing strategies influence willingness-to-pay: anchoring and framing effects. Journal of Travel Research. DOI : http://dx.doi.org/10.1177/0047287518793037

Taylor W et Kimes SE (2011) The effect of brand class on perceived fairness of revenue management. Journal of Revenue and Pricing Management 10(3): 271-284.

Thiery P (2005) Marketing et responsabilité sociétale de 1'entreprise: entre civisme et cynisme. Décisions Marketing 38: 59-69.
Tripathi S (2017) Et tu, Brute? How unfair! Journal of Retailing and Consumer Services 39: 79-92.

Vives A, Jacob M et Payeras M (2018) Revenue management and price optimization techniques in the hotel sector: a critical literature review. Tourism Economics 24(6): 720-752.

Weatherford LR et Bodily SE (1992) A taxonomy and research overview of perishable-asset revenue management: yield management, overbooking, and pricing. Operations Research 40(5): 83-84.

Wu CC, Liu YF, Chen YJ et Wang CJ (2012) Consumer responses to price discrimination: discriminating bases, inequality status, and information disclosure timing influences. Journal of Business Research 65(1): 106-116.

Vukadin A, Lemoine JF et Badot O (2019) Store artification and retail performance. Journal of Marketing Management 35(7-8): 634-661.

Xia L et Monroe KB (2010) Is a good deal always fair? Examining the concepts of transaction value and price fairness. Journal of Economic Psychology 31: 884-894.

Xia L, Monroe KB et Cox JL (2004) The price is unfair! A conceptual framework of price fairness perceptions. Journal of Marketing 68(October): $1-15$.

Zhang X et Jiang B (2014) Increasing price transparency: implications of consumer price posting for consumers' haggling behavior and a seller's pricing strategies. Journal of Interactive Marketing 28(1): 68-85.

Annexe I. Synthèse des définitions des termes employés dans les recherches sur le RM.

\begin{tabular}{|c|c|c|}
\hline Revenue management & Yield management & Pricing ou TRM \\
\hline $\begin{array}{l}\text { Le RM est une stratégie globale de prévision, } \\
\text { d'optimisation et du contrôle des capacités, des } \\
\text { prix et du chiffre d'affaires dans les entreprises } \\
\text { caractérisées par des capacités contraintes } \\
\text { et des actifs périssables (Buckhiester, 201 I; } \\
\text { Weatherford et Bodily, 1992) }\end{array}$ & $\begin{array}{l}\text { Le but du yield } \\
\text { management est la gestion } \\
\text { des recettes unitaires grâce } \\
\text { à une allocation optimale } \\
\text { des capacités par classe } \\
\text { tarifaire (Capiez, 2003) }\end{array}$ & $\begin{array}{l}\text { Le pricing ou la TRM a pour } \\
\text { but d'organiser et de gérer la } \\
\text { politique tarifaire et les grilles } \\
\text { de prix en fonction des objectifs } \\
\text { globaux fixés dans le cadre du RM } \\
\text { (Heo et Lee, } 20 \mathrm{II} \text { ) }\end{array}$ \\
\hline
\end{tabular}

NB: La TRM ou pricing est une composante du revenue management (RM). Le RM ne se résume pas au pricing. II mobilise également d'autres leviers comme la surréservation, la gestion de la distribution, l'analyse des performances, etc. (Kimes, I994).

Annexe 2. L'échelle du CAP de Maxwell (2002) avec sa traduction en français.

Items d'origine en anglais

The likelihood of my purchasing this ticket is. . .

My willingness to buy the ticket is. . .

The probability that I would consider buying

this ticket is. . .
Traduction en français

La probabilité que j'achète ce billet est. . .

Mon intention de payer ce billet est. . .

La probabilité pour moi d'acheter ce billet est. . . 
Annexe 3. Mesure de fiabilité et de validité des variables endogènes et exogènes du modèle.

\begin{tabular}{|c|c|c|c|c|c|}
\hline Variables latentes & Indicateurs réflexifs (items) & Loadings & $\begin{array}{l}\text { Alpha de } \\
\text { Cronbach }(\alpha)\end{array}$ & $\begin{array}{l}\text { Rho de } \\
\text { D.G. }(p)\end{array}$ & AVE \\
\hline \multirow{3}{*}{$\begin{array}{l}\text { Equité perçue du prix } \\
\text { (EPP) }\end{array}$} & Prix proportionnel & 0,90 & \multirow[t]{3}{*}{0,81} & \multirow[t]{3}{*}{0,88} & \multirow[t]{3}{*}{0,71} \\
\hline & Prix impartial & 0,71 & & & \\
\hline & Prix justifié & 0,91 & & & \\
\hline \multirow{3}{*}{$\begin{array}{l}\text { Transparence perçue } \\
\text { de l'information (TPI) }\end{array}$} & Information claire & 0,66 & \multirow{3}{*}{0,65} & \multirow{3}{*}{0,81} & \multirow[t]{3}{*}{0,57} \\
\hline & Explication & 0,70 & & & \\
\hline & Information précise & 0,88 & & & \\
\hline \multirow{5}{*}{$\begin{array}{l}\text { Dimension cognitive de } \\
\text { l'injustice perçue }(\mathrm{DCl})\end{array}$} & Ces prix sont inacceptables & 0,89 & \multirow[t]{5}{*}{0,89} & \multirow[t]{5}{*}{0,92} & \multirow[t]{5}{*}{0,70} \\
\hline & Ces prix sont malhonnêtes & 0,83 & & & \\
\hline & Ces prix choquants & 0,85 & & & \\
\hline & Ces prix sont incompréhensibles & 0,82 & & & \\
\hline & Ces prix ne sont pas logiques & 0,79 & & & \\
\hline \multirow{3}{*}{$\begin{array}{l}\text { Dimension affective de } \\
\text { l'injustice perçue (DCl) }\end{array}$} & On est pris pour dupe & 0,79 & \multirow[t]{3}{*}{0,74} & \multirow[t]{3}{*}{0,85} & \multirow[t]{3}{*}{0,66} \\
\hline & On se moque de nous & 0,81 & & & \\
\hline & On est manipulé & 0,83 & & & \\
\hline \multirow{3}{*}{$\begin{array}{l}\text { Consentement à payer } \\
\text { (CAP) }\end{array}$} & J'accepte ces prix & 0,84 & \multirow[t]{3}{*}{0,74} & \multirow[t]{3}{*}{0,85} & \multirow[t]{3}{*}{0,65} \\
\hline & Je suis prêt à payer ces prix & 0,81 & & & \\
\hline & Je suis d'accord pour payer ces prix & 0,78 & & & \\
\hline
\end{tabular}

Annexe 4. Présentation du plan factoriel et des stimuli de la recherche

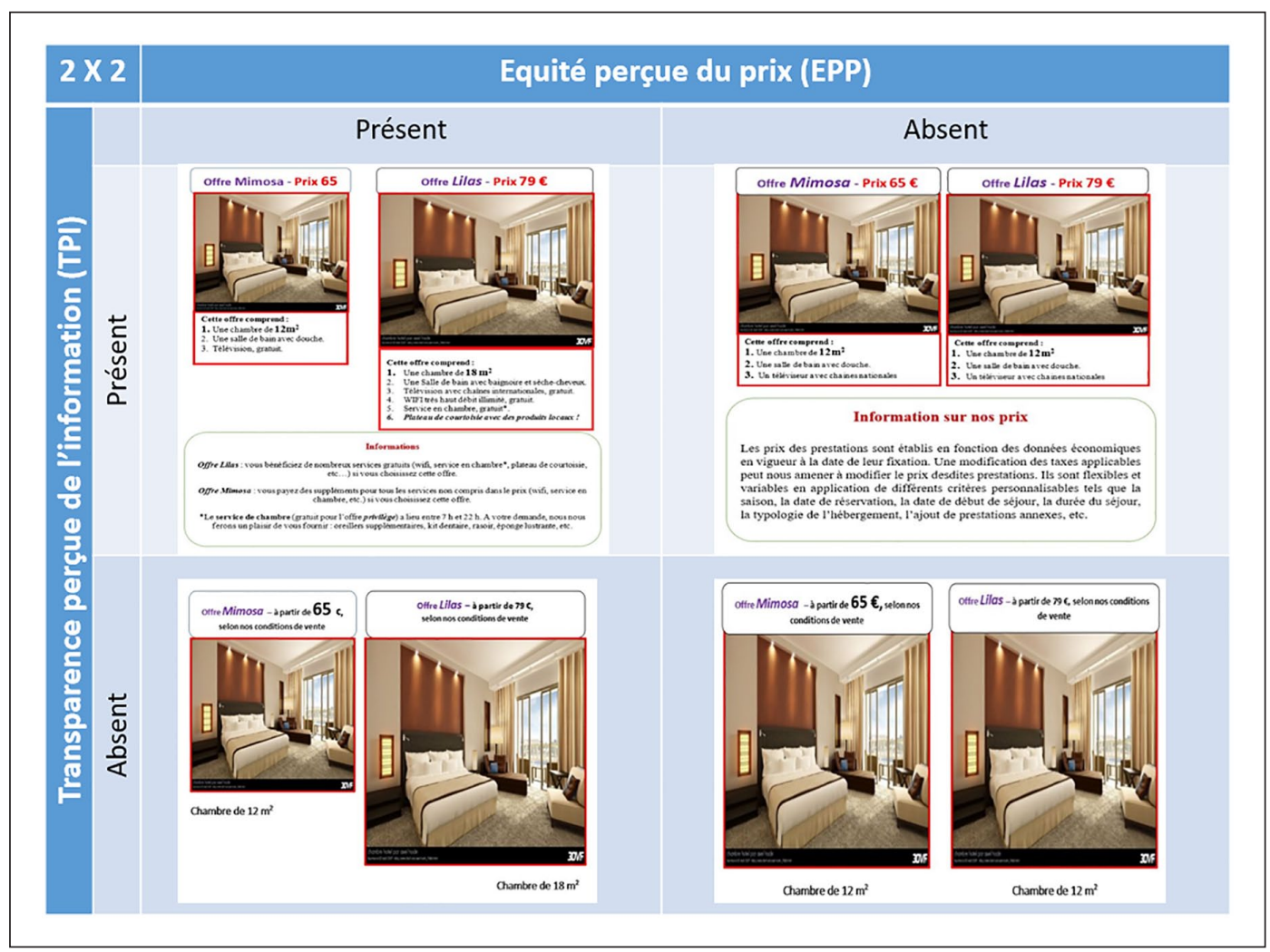


Dans la première condition (scénario 1), l'équité du prix et la transparence de l'information sont simultanément testées afin de mesurer les effets d'interaction. Dans la deuxième condition (scénario 2), seule la transparence est testée afin de mesurer ses effets individuels. Dans la troisième condition (scénario 3), seule l'équité est testée. Enfin, dans la quatrième condition (scénario 4), l'équité et la transparence ont été conjointement supprimées afin de mesurer les effets de leur absence conjointe sur les variables dépendantes.

Annexe 5. Récapitulatif des scores des variables latentes du modèle I.

I. Scores des effets individuels de l'équité perçue (EPP) et de la transparence perçue (TPI) sur la dimension cognitive de l'injustice perçue $(\mathrm{DCl})$.

\begin{tabular}{llllll}
\hline $\mathrm{R}^{2}(\mathrm{DCl} / \mathrm{I})$ & $\mathrm{F}$ & $\mathrm{Pr}>\mathrm{F}$ & $\mathrm{R}^{2}$ (Bootstrap) & Erreur standard & Ratio critique \\
\hline 0,68 & 289,23 & 0,00 & 0,69 & 0,06 & 10,55 \\
$\begin{array}{l}\text { Path coefficients }(\mathrm{DCl} / \mathrm{I}): \\
\text { Variable latente }\end{array}$ & Valeur & Erreur standard & $\mathrm{t}$ & $\mathrm{Pr}>|\mathrm{t}|$ & $\mathrm{f}^{2}$ \\
\hline EPP & 0,76 & 0,03 & 21,70 & 0,00 & 1,70 \\
TPI & 0,20 & 0,03 & 5,66 & 0,00 & 0,12 \\
\hline
\end{tabular}

2. Scores des effets individuels de l'équité perçue (EPP) et de la transparence perçue (TPI) sur la dimension affective de l'injustice perçue (DAl).

\begin{tabular}{llllll}
\hline $\mathrm{R}^{2}(\mathrm{DAl} / \mathrm{I})$ & $\mathrm{F}$ & $\mathrm{Pr}>\mathrm{F}$ & $\mathrm{R}^{2}$ (Bootstrap) & Erreur standard & Ratio critique \\
\hline 0,16 & 26,27 & 0,00 & 0,22 & 0,08 & 2,00 \\
Path coefficients $(\mathrm{DAl} / \mathrm{I}):$ & & & & \\
\hline Variable latente & Valeur & Erreur standard & $\mathrm{t}$ & $\mathrm{Pr}>|\mathrm{t}|$ & $\mathrm{f}^{2}$ \\
\hline EPP & $-0,38$ & 0,06 & $-6,68$ & 0,00 & 0,16 \\
TPI & 0,23 & 0,06 & 4,14 & 0,00 & 0,06 \\
\hline
\end{tabular}

3. Scores des effets individuels de l'équité perçue (EPP) et de la transparence perçue (TPI) sur le consentement à payer (CAP).

\begin{tabular}{lcllll}
\hline $\mathrm{R}^{2}(\mathrm{CAP} / \mathrm{I})$ & $\mathrm{F}$ & $\mathrm{Pr}>\mathrm{F}$ & $\mathrm{R}$ (Bootstrap) & Erreur standard & Ratio critique \\
\hline $\begin{array}{l}\text { 0,7I } \\
\text { Path coefficients }(\mathrm{CAP} / \mathrm{I}):\end{array}$ & & 0,00 & 0,06 & $\mathrm{II}, 46$ \\
\hline Variable latente & Valeur & Erreur standard & $\mathrm{t}$ & $\mathrm{Pr}>|\mathrm{t}|$ & $\mathrm{f}$ \\
\hline EPP & $0,8 \mathrm{I}$ & 0,03 & 24,72 & 0,00 & $2,2 \mathrm{I}$ \\
TPI & 0,12 & 0,03 & 3,58 & 0,00 & 0,05 \\
\hline
\end{tabular}


Annexe 6. Récapitulatif des scores des variables latentes du modèle 2.

I. Scores des effets d'interactions entre l'équité perçue (EPP) et de la transparence perçue (TPI) sur la dimension cognitive de l'injustice perçue (DCl).

\begin{tabular}{llllll}
\hline $\mathrm{R}^{2}(\mathrm{DCl} / 2)$ & $\mathrm{F}$ & $\mathrm{Pr}>\mathrm{F}$ & $\mathrm{R}$ (Bootstrap) & Erreur standard & Ratio critique \\
\hline $\begin{array}{l}\text { 0,69 } \\
\text { Path coefficients }(\mathrm{DCl} / 2):\end{array}$ & 608,27 & 0,00 & 0,70 & 0,06 & $\mathrm{II}, 14$ \\
\hline Variable latente & Valeur & Erreur standard & $\mathrm{t}$ & $\mathrm{Pr}>|\mathrm{t}|$ & $\mathrm{f}$ \\
\hline EPP $\times$ TPI & 0,83 & 0,03 & 24,66 & 0,00 & 2,19 \\
\hline
\end{tabular}

2. Scores des effets d'interactions entre l'équité perçue (EPP) et de la transparence perçue (TPI) sur la dimension affective de l'injustice perçue (DAl).

\begin{tabular}{llllll}
\hline $\mathrm{R}^{2}(\mathrm{DAl} / 2)$ & $\mathrm{F}$ & $\mathrm{Pr}>\mathrm{F}$ & $\mathrm{R}$ (Bootstrap) & Erreur standard & Ratio critique \\
\hline $\begin{array}{l}0,20 \\
\text { Path coefficients (DAl /2): }\end{array}$ & 0,00 & 0,23 & 0,09 & 2,27 \\
\hline Variable latente & Valeur & Erreur standard & $\mathrm{t}$ & $\mathrm{Pr}>|\mathrm{t}|$ & $\mathrm{f}$ \\
\hline EPP $\times$ TPI & $-0,45$ & 0,05 & $-8,30$ & 0,00 & 0,25 \\
\hline
\end{tabular}

3. Scores des effets d'interactions entre l'équité perçue (EPP) et de la transparence perçue (TPI) sur le consentement à payer (CAP).

\begin{tabular}{llllll}
\hline $\mathrm{R}^{2}(\mathrm{CAP} / 2)$ & $\mathrm{F}$ & $\mathrm{Pr}>\mathrm{F}$ & $\mathrm{R}$ (Bootstrap) & Erreur standard & Ratio critique (CR) \\
\hline $\begin{array}{l}\text { 0,70 } \\
\text { Path coefficients }\end{array}$ & $\begin{array}{l}\text { 662,I5 } / 2): \\
\text { Variable latente }\end{array}$ & 0,00 & $0,7 \mathrm{I}$ & 0,06 & $\mathrm{II}, \mathrm{I2}$ \\
\hline Valeur & Erreur standard & $\mathrm{t}$ & $\mathrm{Pr}>|\mathrm{t}|$ & $\mathrm{f}$ \\
\hline EPP $\times$ TPI & 0,84 & 0,03 & 25,73 & 0,00 & 2,38 \\
\hline
\end{tabular}

